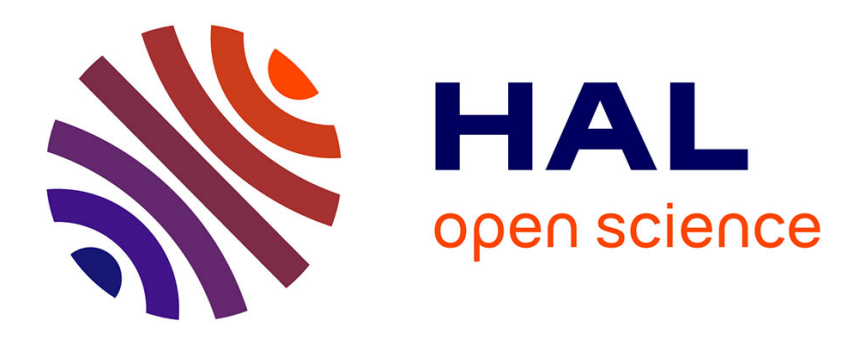

\title{
Poincaré's Equations for Cosserat Media: Application to Shells
}

\author{
Frédéric Boyer, Federico Renda
}

\section{To cite this version:}

Frédéric Boyer, Federico Renda. Poincaré's Equations for Cosserat Media: Application to Shells. Journal of Nonlinear Science, 2016, pp.1-44. 10.1007/s00332-016-9324-7 . hal-01230677v3

\section{HAL Id: hal-01230677 \\ https://hal.science/hal-01230677v3}

Submitted on 3 Oct 2016

HAL is a multi-disciplinary open access archive for the deposit and dissemination of scientific research documents, whether they are published or not. The documents may come from teaching and research institutions in France or abroad, or from public or private research centers.
L'archive ouverte pluridisciplinaire HAL, est destinée au dépôt et à la diffusion de documents scientifiques de niveau recherche, publiés ou non, émanant des établissements d'enseignement et de recherche français ou étrangers, des laboratoires publics ou privés. 


\title{
Poincaré's equations for Cosserat media : application to shells
}

\author{
Frederic Boyer ${ }^{1}$ Federico Renda ${ }^{2}$
}

\begin{abstract}
In 1901 Henri Poincaré discovered a new set of equations for mechanics. These equations are a generalization of Lagrange's equations for a system whose configuration space is a Lie group which is not necessarily commutative. Since then, this result has been extensively refined through the Lagrangian reduction theory. In the present contribution, we apply an extended version of these equations to continuous Cosserat media, i.e. media in which the usual point particles are replaced by small rigid bodies, called micro-structures. In particular, we will see how the Shell balance equations used in nonlinear structural dynamics, can be easily deduced from this extension of the Poincaré's result. In future, these results will be used as foundations for the study of squid locomotion, which is an emerging topic relevant to soft robotics.
\end{abstract}

\section{Acknowledgment}

The final publication is available at:

Boyer, F. \& Renda, F. J Nonlinear Sci (2016). doi:10.1007/s00332-016-9324-7

\footnotetext{
${ }^{1}$ Frédéric Boyer EMN, IRCCyN, La Chantrerie 4, rue Alfred Kastler B.P. 20722 - 44307 Nantes Cedex 3 France. Tel.: (33)02.51.85.83.08, Fax: (33)02.51.85.83.40 , E-Mail: frederic.boyer@emn.fr

${ }^{2}$ Federico Renda KUSTAR, KURI, Abu Dhabi campus, 127788 Abu Dhabi, UAE. Tel: +971 (0) 2 4018082, Fax: +971 (0) 2 4472442, Email: federico.renda@kustar.ac.ae
} 


\title{
Nomenclature
}

\author{
$t \quad$ time \\ $\mathcal{E} \quad 3$-dimensional geometric space of classical mechanics \\ $\mathcal{B} \quad 3$-dimensional material space of a classical continuous medium \\ $\mathcal{D} \quad$ Material $(p$-dimensional, $p<3)$ reference subspace \\ $\mathcal{M}$ Rigid microstructure \\ $\mathcal{B}=\mathcal{D} \times \mathcal{M}$ Material space of a Cosserat medium \\ $\left(O, E_{1}, E_{2}, E_{3}\right)$ Material frame attached to $\mathcal{B}$ \\ $\left(o, e_{1}, e_{2}, e_{3}\right)$ Spatial frame attached to $\mathcal{E}$ \\ $x=x^{i} e_{i}$ Points of geometric space \\ $X=X^{i} E_{i}$ Material points of $\mathcal{B}$ \\ $\bar{X}=X^{\alpha} E_{\alpha}$ Material points of $\mathcal{D}$ \\ $\Phi_{t} \quad$ Transformation at time $t$ from material to geometric space \\ $\Phi_{t}(\mathcal{B})$ Deformed configuration of $\mathcal{B}$ \\ $\Phi_{o}(\mathcal{B})$ Reference configuration of $\mathcal{B}$ \\ $\left(\Phi_{t} \circ e\right)(\mathcal{D})$ Deformed configuration of $\mathcal{D}$ \\ $\left(\Phi_{o} \circ e\right)(\mathcal{D})$ Reference configuration of $\mathcal{D}$ \\ $r(\bar{X}) \quad$ Position of $\left(\Phi_{t} \circ e\right)(\bar{X})$ \\ $R(\bar{X}) \in S O(3)$ Rotation tensor mapping $\left(E_{1}, E_{2}, E_{3}\right)$ onto $\left(t_{1}, t_{2}, t_{3}\right)(\bar{X})$ \\ $\left(g_{1}, g_{2}, g_{3}\right)(X)$ Convected basis on $\Phi_{t}(\mathcal{B})$ at $\Phi_{t}(X)$ \\ $\left(h_{1}, \ldots h_{p}\right)(\bar{X})$ Convected basis on $\left(\Phi_{t} \circ e\right)(\mathcal{D})$ at $r(\bar{X})$ \\ $\left(t_{1}, t_{2}, t_{3}\right)(\bar{X})$ Orthonormal spatial basis attached to the $X$-microstructure \\ $\left(g_{i j} g^{i} \otimes g^{j}\right)(X)$ Euclidean metric tensor in the convected basis of $\Phi_{t}(\mathcal{B})$ \\ $\left(h_{\alpha \beta} h^{\alpha} \otimes h^{\beta}\right)(\bar{X})$ Euclidean metric induced on $\left(\Phi_{t} \circ e\right)(\mathcal{D})$ in its convected basis \\ $\nu, \nu_{o}, \nu_{t}$ Oriented unit normal vector to the material, reference and deformed \\ surface element of $\mathcal{D}$ \\ $d S, d S_{o}, d S_{t}$ Area of the material, reference and deformed surface element of $\mathcal{D}$ \\ $\mathcal{C} \quad$ Configuration space of a Cosserat medium $\mathcal{D} \times \mathcal{M}$ \\ $G$ and $\mathrm{g}$ Group of transformation and transformation of microstructure \\ $\mathfrak{g}, \mathfrak{g}^{*} \quad$ Lie algebra of $G$ and its dual \\ $A d$ and $A d^{*}$ Adjoint and coadjoint action map of $G$ on $\mathfrak{g}$ and $\mathfrak{g}^{*}$ \\ $a d$ and $a d^{*}$ Adjoint and coadjoint action map of $\mathfrak{g}$ on $\mathfrak{g}$ and $\mathfrak{g}^{*}$ \\ $\eta$ and $\xi_{\alpha}$ Left invariant fields along time and space-variables \\ $\mathfrak{L}, \mathfrak{L}_{o}$ and $\mathfrak{L}_{t}$ Density of left-reduced Lagrangian of a Cosserat medium per unit \\ of its material, reference and deformed volume \\ $\frac{\partial \mathfrak{L}}{\partial \eta}, \frac{\partial \mathfrak{L}_{o}}{\partial \eta}$ and $\frac{\partial \mathfrak{L}_{t}}{\partial \eta}$ Densities of material $t$-conjugate (kinetic) momentum, per unit \\ of material, reference and deformed volume \\ $\frac{\partial \mathfrak{L}}{\partial \xi_{\alpha}}, \frac{\partial \mathfrak{L}_{o}}{\partial \xi_{\alpha}}$ and $\left[\frac{\partial \mathfrak{L}}{\partial \xi_{\alpha}}\right]_{t}$ Densities of material $X^{\alpha}$-conjugate (stress) momentum, \\ per unit of material, reference and deformed volume \\ $F_{e x t}, F_{e x t, o}$ and $F_{e x t, t}$ Densities of material external forces per unit of material, \\ reference and deformed volume \\ $\bar{F}_{e x t}, \bar{F}_{e x t, o}$ and $\bar{F}_{e x t, t}$ Densities of external forces per unit of material, refer- \\ ence, deformed boundary volume
}


$\mathcal{D} \times \mathbb{R}^{+}$Space-time of a $p$-dimensional Cosserat medium

$X^{0} \frac{\partial}{\partial t}+X^{\alpha} \frac{\partial}{\partial X^{\alpha}}$ Point in space-time with $t=X^{0}$

$\Upsilon \quad$ Space-time 1-form field with value in $\mathfrak{g}$

$\Lambda, \Lambda_{o}$ and $\Lambda_{t}$ Density of a space-time vector field with value in $\mathfrak{g}^{*}$, per unit of material, reference, deformed volume

$<., .>$ and $(.,$.$) Duality product in \mathfrak{g}$ and space-time

$A d_{\mathrm{g}^{-1}}^{*}\left(\sqrt{|h|}\left(\frac{\partial \mathfrak{L}_{t}}{\partial \eta}\right)\right)$ Densities of spatial (in the fixed frame) kinetic wrench, per

$A d_{\mathrm{g}^{-1}}^{*}\left(\sqrt{|h|}\left(\frac{\partial \mathfrak{L}_{t}}{\partial \xi_{\alpha}}\right)\right)$ Densities of spatial (in the fixed frame) stress wrench, per unit of deformed volume

$S E(3)$ Special Euclidean Group in $\mathbb{R}^{3}$ with Lie algebra $s e(3)$

$(R, r)$ Transformation of $S E(3)$

$\left(\Omega^{T}, V^{T}\right)^{T} \in \operatorname{se}(3)$ Material time-rate of transformation (velocity) of the microstructure frames

$\left(\omega^{T}, v^{T}\right)^{T} \in s e(3)$ Spatial time-rate of transformation (velocity) of the microstructure frames

$\left(\Sigma_{t}^{T}, P_{t}^{T}\right)^{T} \in s e(3)^{*}$ Density of material kinetic wrench per unit of deformed volume

$\left(\sigma_{t}^{T}, p_{t}^{T}\right)^{T} \in \operatorname{se}(3)^{*}$ Density of spatial (in the microstructure frame) kinetic wrench per unit of deformed volume

$\left(K_{\alpha}^{T}, \Gamma_{\alpha}^{T}\right)^{T} \in \operatorname{se}(3)$ Material $X^{\alpha}$-rate of transformation of the microstructure frames

$\left(k_{\alpha}^{T}, \gamma_{\alpha}^{T}\right)^{T} \in \operatorname{se}(3)$ Spatial $X^{\alpha}$-rate of transformation of the microstructure frames $\left(M_{\alpha, t}^{T}, N_{\alpha, t}^{T}\right)^{T} \in \operatorname{se}(3)^{*}$ Density of material stress wrench per unit of deformed volume

$\left(m_{\alpha, t}^{T}, n_{\alpha, t}^{T}\right)^{T} \in s e(3)^{*}$ Density of spatial stress wrench per unit of deformed volume

$\left(\bar{\rho}, \bar{\rho}_{o}, \bar{\rho}_{t}\right)$ and $\left(\bar{J}, \bar{J}_{o}, \bar{J}_{t}\right)$ Densities of mass and of material angular inertia tensor per unit of material, reference, deformed volume

$\left(\bar{I}, \bar{I}_{o}, \bar{I}_{t}\right)$ Densities of spatial inertia tensor per unit of material, reference, deformed volume

$\epsilon_{\alpha \beta}, \rho_{\alpha \beta}, \tau_{\alpha}$ Effective strain measures (stretching, bending, transverse shearing) of a classical shell

$\mathcal{N}_{t}^{\alpha \beta}, \mathcal{M}_{t}^{\alpha \beta}, \mathcal{Q}_{t}^{\alpha}$ Densities of effective stress of a classical shell per unit of deformed volume

\section{Introduction}

In contrast to classical continuous media where the basic constitutive element of matter is the point particle, Cosserat media are defined by small rigid bodies, called micro-structures, continuously stacked along material dimensions [1]. This fundamental difference has strong consequences on the two theories (classical vs Cosserat). In the classical theory, the geometric model of finite rotations disappears from the model, only re-appearing as a kinematic consequence of the translations (e.g. through the curl of the linear velocity field), while in the 
Cosserat model the rotations have a status similar to that of translations from the beginning to the end of the dynamic formulation. As a result, the Lie group structure naturally appears in the intrinsic definition of the configuration space of a Cosserat medium through the rigid transformations (in $S O(3)$, and more generally $S E(3)$ ) undergone by its constitutive micro-structures. Thus, the model of Cosserat media should be recoverable from the abstract variational calculus developed by Henri Poincaré [2, known today as the Poincaré or Euler-Poincaré equations [3, [4. These equations can be considered as a generalization of Lagrange's equations to systems whose configuration space is defined as a non commutative Lie group. As Poincaré remarked himself, they are particularly relevant when the Lagrangian of the system is left (or right) invariant by the group transformations, a property which is related to the symmetry of space (left invariance) and matter (right invariance) as Arnold and Marsden discovered later through the Lagrangian reduction theory [3, [4]. In this context, the right invariance has been shown to be a key concept to include the Eulerian point of view of fluid mechanics within the Euler-Poincaré's approach [5]. Arnold demonstrated that the ideal fluid is the infinite (right invariant) counterpart of the finite (left invariant) Euler and Poincaré's rigid body [6. Another case that motivates us to shift from the finite to the infinite dimensional case is that of Cosserat media [7. While in the case of the ideal fluid, the transformations live in an infinite dimensional group, in the case of Cosserat media the transition toward infinite dimension is dramatically different. In this other case, in each point of a continuous material medium $\mathcal{D}$, a finite dimensional group $G$ acts on a microstructure $\mathcal{M}$, i.e., a rigid body of infinitesimal size. Applying Poincaré's variational calculus to this context requires a shift from the basic picture of ordinary differential Poincaré equations of classical mechanical systems, to a set of partial differential equations in a field theoretical approach as it has been developed in the seminal works [8] and [9]. Going back to the original aim of the Cosserat brothers [7, in [8], the fields equations of an unbounded Cosserat medium are derived in the context of the abstract formal theory of systems of partial differential equations [10, with no reference to the Poincaré picture. From a more geometrical viewpoint, in [9, the original variational calculus of Poincaré is extended from a configuration Lie group to a principal fiber bundle to derive a set of covariant Euler-Poincaré equations but with no relation to Cosserat media. In [11, both fields equations and boundary conditions of a bounded multidimensional Cosserat medium are derived in the context of Euler-Poincaré reduction. In this approach, the dynamics of the Cosserat medium are deduced from a unique Lagrangian density left invariant by the transformations of $G$, the transformations being parameterized by the time and the material coordinates (Lagrangian labels) of the medium $\mathcal{D}$. Though it may seem abstract at first, this variational calculus, which generalises Poincaré's calculus from one parametric dimension (the time axis) to several (space-time), is in fact a powerful alternative tool to Newton's laws and Euler's theorems for deriving in a blind manner the balance equations of Cosserat media. Furthermore, revealing the intrinsic geometric nature of these media, the approach can assist in the development of numerical methods able to cope with 
finite rotations. In this later context, Cosserat media have been promoted in the field of the Finite Element Method, under the name of the "geometrically exact approach" by J.C. Simo and co-authors [12, [13, but with no reference to the Euler-Poincaré reduction theory. In particular, though the Hamiltonian structure of the geometrically exact balance equations of rods and plates is revealed in 14 through the derivation of an appropriate bracket, these equations are considered as a starting point in [14, and derived from Newton's laws and Euler's theorems. More recently, the relations between Lagrangian reduction and geometrically exact beam theory, have been established and exploited in [11, [15] and [16, with further extension to the case of molecular strands [17, [18. In [18, several sets of reduced motion equations, ranging from Euler-Poincaré to Lagrange-Poincaré equations, are developed for modelling molecular strands subjected to nonlocal electrostatic forces, while in the same reference, the case of multidimensional media (in this case, molecular membranes), is evoked as a further perspective by the authors. In the robotics community, the Poincaré equations for Cosserat beams have also raised a growing interest by proving that they are an efficient tool to model the locomotion of novel continuous (hyperredundant) and soft robots inspired from fish [19, snakes and worms [20, as well as the manipulation by soft robots inspired from octopus arm 21. More recently, cephalopods have drawn the attention of bioroboticists with the long term aim of designing squid-like swimming robots able to propel by cyclically contracting a soft shell cavity inspired from the mantle of these animals 22 .

Originally driven by the need to model soft robots inspired from squids, the present article aims at applying the Poincare picture to the case of multidimensional Cosserat media and especially, the Cosserat shells. To that end, we will restart from the general construction of [11, and will remind how one can derive from a Lagrangian density related to the space of material labels of $\mathcal{D}$, a first set of Poincaré equations that will be consequently named "Poincaré equations of Cosserat media in the space of material labels", or more concisely, "in the material space $\mathcal{D}^{\prime \prime}$. In this general context, we will consider both the field equations and the boundary conditions of a Cosserat medium $(\mathcal{D}, \mathcal{M})$, with $\mathcal{D}$ of arbitrary dimension $p$, and $\mathcal{M}$ a full (non-degenerated) three-dimensional (3D) microstructure. This first set of equations being related to $\mathcal{D}$, i.e., to a space disconnected from the physical geometric space, it is physically inconsistent and practically unusable for shells. As a result, we will need to derive two further sets of Poincaré equations, one related to the reference configuration, and the second, related to the deformed (current) configuration of the medium, both being embedded in geometric space. To derive these new equations, we will start from the equations in the material space $\mathcal{D}$ of [11]. Then, lying on the concept of duality, we will identify the intrinsic geometric nature of all the objects they handle, and establish how they transform from material space $\mathcal{D}$ to the reference and deformed configuration of the medium. In parallel to this first approach of derivation, we will show that these two sets of equations can be derived straightforwardly by extending the variational calculus of [11] to Lagrangian densities 
related to reference and deformed configurations of the Cosserat medium. Along derivation of these equations, we will progressively relate the objects naturally produced by the Poincaré calculus to the physics of the Cosserat media, and will recover in a pure deductive manner, several of the key concepts of the micropolar theory [1, as those related to the material objectivity of their constitutive laws, their kinematic and kinetic models, several models of strain and stress, and their balance equations in the geometrically exact form. At the end, the approach will give a structured picture of the model of Cosserat media, while opening promising perspectives for future.

For the purpose of illustration, we will then show how these further general formulations in geometric space, allow recovering the so called geometrically exact balance equations of the existing shell theory as they have been developed over the years by other means in works by Reissner [23], Green and Naghdi [24], Antman [25], Libai [26] among others. Applying our general equations in the case $p=2$, will give the geometric exact balance equations of a micropolar shell [26], with several modalities of expression depending on whether they are related to the reference or current configuration of $\mathcal{D}$, and, in the material frame or the spatial frame of $\mathcal{M}$. Based on these nominal sets of equations, we will shift from micropolar to classical shell, while stressing the role of the constitutive laws in a generic reduction process allowing to recover the geometrically exact formulation of classical shells in which the microstructure degenerate into a director [23],24, 225, [12]. Following [12], in the resulting reduced dynamic model, the angular velocity field around the directors, referred as the "drilling rotation" in the shell literature [27, is arbitrarily forced to zero. In a final step, we will reincorporate the drilling degrees of freedom and derive a further closed formulation holding for classical Cosserat shells with drilling rotations. While this approach is inspired of works in finite-elements [28, it is here stated in a new form which exploits the state-space form of the Poincare picture, as a functional space of surfaces (parameterized by the labels of $\mathcal{D}$ ) in $S E(3) \times s e(3)$.

The article is structured as follows. Section 2 introduces all the basic definitions and statements required by the extension of the Poincaré picture to a Cosserat medium of arbitrary dimension. In section 3, we derive the Poincaré equations of a Cosserat medium in the space of its material labels. Section 4 reconsider these first equations from the viewpoint of duality and analyses the intrinsic geometric nature of the objects they handled, and how they transform from the material to the geometric space. Based on this analysis, in section 5 , the Poincaré equations of $p$-dimensional Cosserat medium in the reference and deformed configuration are derived, and their underlaying model of stress is detailed in relation to continuous media mechanics. In section 6 , this general picture is applied to 2-dimensional Cosserat media to derive the geometrically exact balance equations of micropolar shells. From these first set of equations, we deduce to other sets of equations, those of classical shells with no model of the drilling rotations (section 7), and with a model of the drilling rotations (section 8). For the pur- 
pose of illustration, the full Poincaré picture is applied from its beginning to axisymetric shells in section 9. Section 10 summarizes and opens perspectives for applications to squid locomotion in the context of soft Robotics.

\section{Basic statements and definitions}

In this section we state the basic definitions required for the rest of the article. We invite the reader who is familiar with the geometric point of view of finite elasticity [29] to go directly to subsection 2.5. The key information of sections 2.1-4 are essentially: the definition of the configuration space and the basic kinematics of a Cosserat medium (eq. (3) and (8)), the expressions of the area element in the deformed configuration (eq. (12)), along with the figure 1 which illustrates the geometric context used in the article.

\subsection{Definition and space of configuration of a classical medium}

According to the mechanics of continuous media, a classical three-dimensional medium $\mathcal{B}$ is a compact set of material points of Euclidean space $\mathbb{R}^{3}$ labelled by 3 parameters $\left\{X^{i}\right\}_{i=1,2,3}$ in a Cartesian frame $\left(O, E_{1}, E_{2}, E_{3}\right)$ named material frame. A configuration of $\mathcal{B}$ is the definition of the position $x=x^{i}\left(X^{j}\right) e_{i}$ of all the material points $X=X^{j} E_{j}$ of $\mathcal{B}$ in an inertial frame $\left(o, e_{1}, e_{2}, e_{3}\right)$ of the ambient Euclidean space $\mathcal{E} \cong \mathbb{R}^{3}$. Formally, we define such a configuration as $\Phi(\mathcal{B})$ (this is the set of the $x=\Phi(X)$ for $X$ running over $\mathcal{B})$, where $\Phi$ is a smooth invertible map from $\mathbb{R}^{3}$ to $\mathbb{R}^{3}$ which preserves orientation, i.e., an element of $\operatorname{Diff}\left(\mathbb{R}^{3}\right)$. The space of configurations of $\mathcal{B}$ in $\mathcal{E}$ is thus defined as:

$$
C=\left\{\Phi(\mathcal{B}) \subset \mathcal{E}, \forall \Phi \in \operatorname{Diff}\left(\mathbb{R}^{3}\right)\right\},
$$

and a motion of $\mathcal{B}$ in $\mathcal{E}$ is defined as a curve of configuration, i.e., a mapping:

$$
t \in \mathbb{R}^{+} \mapsto \Phi_{t}(\mathcal{B}) \subset \mathcal{E}
$$

where $\Phi_{t}$ denotes a time-parameterized curve of $\operatorname{Diff}\left(\mathbb{R}^{3}\right)$. Among all the possible configurations accessible to $\mathcal{B}$, we distinguish one of them as a reference configuration, denoted $\Phi_{o}(\mathcal{B})$ in which $\mathcal{B}$ is internally (energetically) at rest ${ }^{3}$. In practise we will assume $\left(O, E_{1}, E_{2}, E_{3}\right)=\left(o, e_{1}, e_{2}, e_{3}\right)$ and will interchangeably speak about the "inertial" or "material frame", depending on the context. For the purpose of computation, one may consider in all subsequent developments that $E_{1}=(1,0,0)^{T}, E_{2}=(0,1,0)^{T}$, and $E_{3}=(0,0,1)^{T}$. Finally, note that these definitions can be extended from three, to two, and one-dimensional classical media, with a material index $i$ running from 1 to $n$ with $n=2$ and $n=1$ respectively. Moreover, it is worth noting that $\left\{X^{i}\right\}_{i=1, \ldots n}$ defines a (material) chart

\footnotetext{
${ }^{3}$ Here note that $\Phi$ is not a mechanical transformation of the body in the ambient space but rather a geometric transformation or more exactly a parameterization of $\mathcal{B}$. The mechanical transformation between the reference and the deformed configurations is in fact defined as $\Phi_{t} \circ \Phi_{o}^{-1}$.
} 
on the open set $\mathcal{B}-\partial \mathcal{B}$, and that for topological reasons, it may be convenient to provide $\mathcal{B}$ with an atlas of several such material charts, even if, in the following, we will consider only one of them.

\subsection{Definition and space of configuration of a Cosserat medium}

A Cosserat medium $(\mathcal{D}, \mathcal{M})$ is a classical $p$-dimensional $(p \leq 3)$ medium $\mathcal{D}$, in each point, noted $\bar{X}$, of which, a Lie group $G$ of rigid body mechanics $(S O(3)$, $S E(2), S E(3) \ldots)$ acts on a rigid solid of small dimensions (a "micro-solid" or "microstructure"), denoted $\mathcal{M}$, to generate all the possible configurations of $(\mathcal{D}, \mathcal{M})$. The configuration space of a Cosserat medium $(\mathcal{D}, \mathcal{M})$ can thus be defined as the following set of parameterized maps in $G$ :

$$
\mathcal{C}=\{\mathrm{g}: \bar{X} \in \mathcal{D} \mapsto \mathrm{g}(\bar{X}) \in G\} .
$$

Motions of $(\mathcal{D}, \mathcal{M})$ in $\mathcal{C}$ are defined as the time-parameterized curves of configuration:

$$
t \in \mathbb{R}^{+} \mapsto \mathrm{g}_{t}(\mathcal{D})=\mathrm{g}(\mathcal{D}, t) \subset G .
$$

where the notation $\mathrm{g}(\mathcal{D}, t)$ indicates that the space and time variables play similar roles. This basic context can be used to describe different physical situations depending on the meaning we attribute to $\mathcal{D}$ and $\mathcal{M}$. For instance, a non-classical three dimensional (3D) medium constituted of three-dimensional micro-structures, also called micro-polar medium, obeys this definition if we take $\mathcal{M} \simeq \mathcal{D} \simeq \mathbb{R}^{3}$. In all the article, we will preferentially use the above definition as a reduced model to describe a classical $3 \mathrm{D}$ medium $\mathcal{B}$ for which $\mathcal{B}=\mathcal{D} \times \mathcal{M}$ where $\mathcal{D}$ is a sub-manifold of $\mathcal{B}$ over each point $\bar{X}$ of which, $\mathcal{M}$ is transformed by an element of $G=S E(3)$, represented by an homogeneous transformation of the general form:

$$
\mathrm{g}(\bar{X})=\left(\begin{array}{cc}
R(\bar{X}) r(\bar{X}) \\
0 & 1
\end{array}\right)
$$

with $R(\bar{X}) \in S O(3)$ and $r(\bar{X}) \in \mathbb{R}^{3}$ being the rotation and translation components of $\mathrm{g}$ respectively. Following section 2.1 , the parameterization of $\mathcal{B}=\mathcal{D} \times \mathcal{M}$ is chosen in such a manner that $\mathcal{D}$ and $\mathcal{M}$ are coordinatized by $\left\{X^{\alpha}\right\}_{\alpha=1,2 . . p}$ and $\left\{X^{\gamma}\right\}_{\gamma=p+1, \ldots 3}$ respectively. Hence, any point in $\mathcal{D}$ is intrinsically defined as $\bar{X}=\left(X^{1}, X^{2}, \ldots X^{p}\right)$ and the map $e: \bar{X} \mapsto e(\bar{X})=\left(\bar{X}, 0_{3-p}\right)$, defines an embedding from $\mathcal{D}$ to $\mathcal{B} \simeq \mathbb{R}^{3}$. This embedding allows any configuration of $\mathcal{D}$ in $\mathcal{E}$ to be defined as the submanifold $(\Phi \circ e)(\mathcal{D})$ of $\Phi(\mathcal{B})$. In $\mathcal{E}$, a configuration of the microstructure $\mathcal{M}$ above $\bar{X}$ will be denoted as $\Phi(\bar{X}, \mathcal{M})$ (see figure 1). With this parametrization the reduction of $\mathcal{B}$ into $\mathcal{D} \times \mathcal{M}$ is motivated by considering material media as beams and shells, having dimensions along $\mathcal{D}$ far larger than the others (along $\mathcal{M}$ ), i.e., media for which the $3 \mathrm{D}$ configurations of (1) can be expanded into the following Taylor series in which $\gamma=p+1, . .3$ :

$$
\Phi(X)=\Phi\left(\bar{X}, 0_{3-p}\right)+\frac{\partial \Phi}{\partial X_{\perp}^{\gamma}}\left(\bar{X}, 0_{3-p}\right) X_{\perp}^{\gamma}+o\left(\left\|X_{\perp}\right\|^{2}\right),
$$


with $X_{\perp}=X-e(\bar{X})$ the vector component of $X$ along $\mathcal{M}$. Based on this expansion, the Cosserat based approach consists in reducing the kinematics (6) to its first order approximation with respect to $\left\|X_{\perp}\right\|$ while neglecting the deformations of the material above each $\left(\bar{X}, 0_{3-p}\right)$, a condition which defines the rigid microstructure $\mathcal{M}$. These approximations allow $(6)$ to be rewritten as:

$$
\Phi(X)=r(\bar{X})+t_{\gamma}(\bar{X}) X_{\perp}^{\gamma}
$$

with $\bar{X} \mapsto r(\bar{X})=(\Phi \circ e)(\bar{X})$ the field of position of the material points of $\mathcal{D}$ in $\mathcal{E}$, and $t_{\gamma}(\bar{X})=\left(\left(\partial \Phi / \partial X_{\perp}^{\gamma}\right) \circ e\right)(\bar{X})$ a set of vectors lying in $\Phi(\bar{X}, \mathcal{M})$. Furthermore, $\mathcal{M}$ being rigid, it is always possible to choose its parameterization $\left\{X^{\gamma}\right\}_{\gamma=p+1, . .3}$ such that $t_{\gamma}=R(\bar{X}) . E_{\gamma}$ with $R(\bar{X}) \in S O(3)$, and to rewrite the reduced kinematics (7) as:

$$
\Phi(X)=r(\bar{X})+t_{\gamma}(\bar{X}) X_{\perp}^{\gamma}=r(\bar{X})+R(\bar{X}) \cdot\left(X_{\perp}^{\gamma} E_{\gamma}\right)=r(\bar{X})+R(\bar{X}) \cdot X_{\perp},
$$

which explicitly makes the group transformations $\mathrm{g}(\bar{X}) \in S E(3)$ of 335 appear, with $R(\bar{X})$ defined as the two-point tensor [30]: $R(\bar{X})=t^{i}(\bar{X}) \otimes E_{i}$, and: $r(\bar{X})=$ $r^{i}(\bar{X}) e_{i}$. As in rigid-body mechanics, these transformations act on the material frame $\left(O, E_{1}, E_{2}, E_{3}\right)$ considered as rigidly attached to $\mathcal{M}$. However they do not transform it into a single frame, but into a field of orthonormal mobile frames $\left(t_{1}, t_{2}, t_{3}\right)(\bar{X})=\left(R(\bar{X}) \cdot E_{1}, R(\bar{X}) \cdot E_{2}, R \cdot(\bar{X}) E_{3}\right)$ based in each point $\left(\Phi_{t} \circ e\right)(\bar{X})$ as illustrated in figure 1 . In [11, the reduced kinematics (8) are applied to beams, while in the second part of the article, they are applied to shells with $\mathcal{D} \simeq \mathbb{R}^{2}$ defining the (material) reference shell's mid surface. For a shell, the microstructure $\mathcal{M}$ models a generic rigid fiber across each point of its midsurface $\mathcal{D}$, i.e. a degenerated one-dimensional rigid body named "director" in the shell's literature [24. This is in contrast with beams, where $\mathcal{M}$ stands for a full 3D rigid body modelling the beam cross-sections. As a result, for shells, shifting from (7) to (8), i.e. replacing $t_{3}$ by $R$ in the basic kinematics introduces an indeterminacy in the model of a classical shell which should be removed by reducing its configuration space from $(3)$ to $\left\{\left(r, t_{3}\right): \bar{X} \in \mathcal{D} \mapsto\left(r(\bar{X}), t_{3}(\bar{X})\right) \in\right.$ $\left.\mathbb{R}^{3} \times S^{2}\right\}$. However, we will initally ignore this fact and consider shells as fullCosserat (2D) media with configuration space (3), i.e. media for which $\mathcal{M}$ is a full (non-degenerated) rigid body to which a full 3D-orthonormal frame can be attached. This will allow us to see that $\bar{X} \mapsto R(\bar{X})$ is univocally defined by a dynamic model obtained by applying Poincaré's picture on the configuration space (3). From a physical point of view, such a model holds for micro-polar shells, i.e. 2D-media with intrinsic kinetic spin and couple stress along $t_{3}$ [1]. In a second step, we will see how the model of $2 \mathrm{D}$-full Cosserat media can be adapted, and the indeterminacy on $R$ can be removed, when considering the classical model of shell in which the frame attached to $\mathcal{M}$ is degenerated into a single director.

\subsection{Convected frame and co-frame}

In all the article, we will use convected frames to express the tensor fields related to the mechanical state of $\mathcal{B}$ and $\mathcal{D}$. A convected frame field is de- 


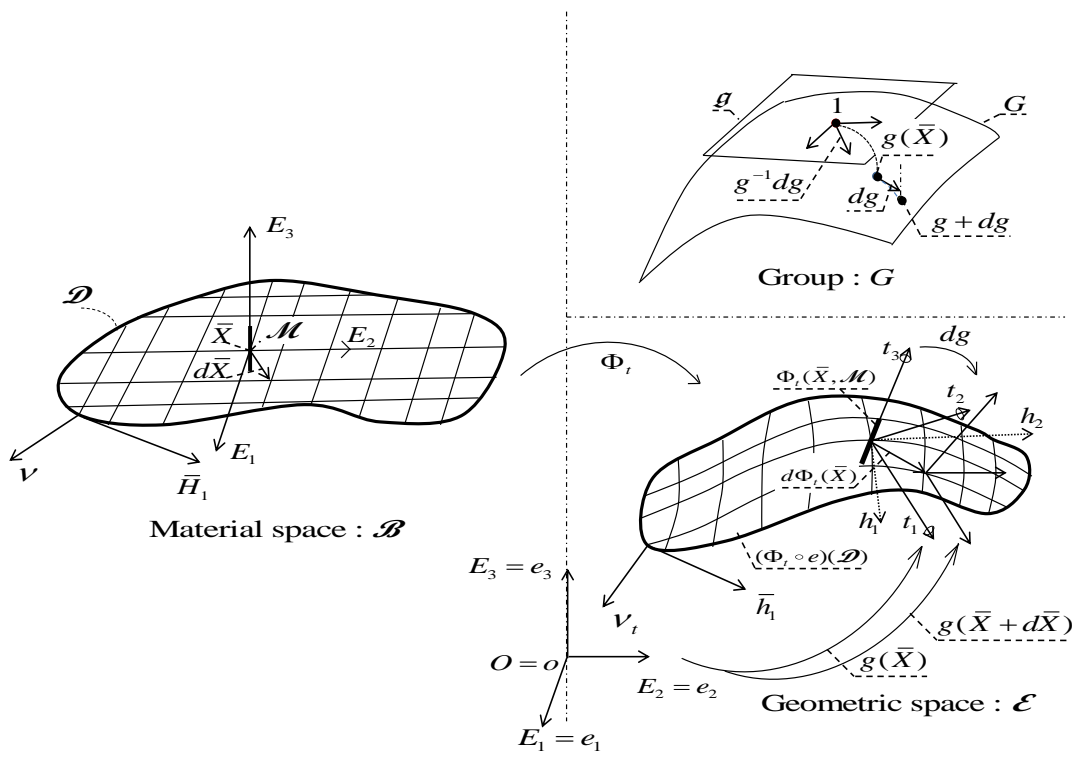

Fig. 1. Left and right bottom: Parameterization of the deformed configuration $\Phi_{t}(\mathcal{B})$ through a transformation of $\mathcal{B}$. Right: Kinematics in $\mathcal{E}$ (bottom) and on $G$ (top) of frames rigidly attached to the micro-structures.

fined in each point of the current configuration of $\mathcal{B}$ as the natural basis tangent to a set of material coordinate lines drawn on $\mathcal{B}$ and advected by its current deformation. Let us consider a motion as defined by (2) with $\Phi_{o}=$ $\Phi_{t=0}$. At any time $t$, we may define the field of the convected frames covering the manifold $\Phi_{t}(\mathcal{B})$ as a map $\Phi_{t}(\mathcal{B}) \mapsto T \Phi_{t}(\mathcal{B})$ which assigns to any point $\Phi_{t}(X) \in \mathcal{E}$, the frame $\left(g_{1}, g_{2}, g_{3}\right)(X)=\left(\left(\partial \Phi_{t} / \partial X^{i}\right)(X)\right)_{i=1,2,3}$. In this field of frame, the Euclidean metric of $\mathcal{E}$ is defined as the fundamental tensor $\left(g_{i} . g_{j}\right)\left(g^{i} \otimes g^{j}\right)=g_{i j}\left(g^{i} \otimes g^{j}\right)$ of determinant $|g|$, where $\Phi_{t}(X) \mapsto\left(g^{1}, g^{2}, g^{3}\right)(X)$ defines a field of co-frame such that $g^{i} \cdot g_{j}=\delta_{j}^{i}$. Applying the same construction to the sub-manifold $\left(\Phi_{t} \circ e\right)(\mathcal{D}) \subset \Phi_{t}(\mathcal{B})$ allows to introduce two other fields of convected frame and co-frame whose base points lie in $\left(\Phi_{t} \circ e\right)(\mathcal{D})$ respectively defined as $\left(h_{\alpha}\right)_{\alpha=1,2 . . p}(\bar{X})=\left(\left(\partial r / \partial X^{\alpha}\right)(\bar{X})\right)_{\alpha=1,2 . . p}$, and $\left(h^{\alpha}\right)_{\alpha=1,2 . . p}(\bar{X})$ such that $h^{\alpha} \cdot h_{\beta}=\delta_{\beta}^{\alpha}$, with $h=h_{\alpha \beta}\left(h^{\alpha} \otimes h^{\beta}\right)$ the fundamental metric tensor on $\left(\Phi_{t} \circ e\right)(\mathcal{D})$ whose determinant is denoted by $|h|$. Using the expression of the reduced kinematics $(7)$ in these definitions, it is straightforward to show that $\left(g_{i}(\bar{X}, 0)\right)_{i=1,2,3}=\left(h_{1}, . . h_{p}, t_{p+1}, . . t_{3}\right)(\bar{X})$. and that, for a onedimensional Cosserat medium (beam), $h_{1}\left(X^{1}\right)$ is a tangent vector to the deformed line of the beam centroids in its material abscissa $X^{1}$, while $\left(t_{2}, t_{3}\right)\left(X^{1}\right)$ span the current configuration of $X^{1}$-cross-sections, i.e., $\Phi_{t}\left(\left(X^{1}\right), \mathcal{M}\right)$. For a 2- 
dimensional Cosserat medium (a shell), $\left(h_{1}, h_{2}\right)\left(X^{1}, X^{2}\right)$ defines a basis of the tangent planes to the shell's mid-surface in the base point of material coordinates $\left(X^{1}, X^{2}\right)$ while $\left(t_{3}\right)\left(X^{1}, X^{2}\right)$ span the current configuration of $\left(X^{1}, X^{2}\right)$-fibers, i.e., $\Phi_{t}\left(\left(X^{1}, X^{2}\right), \mathcal{M}\right)$.

\subsection{Pull-back and push-forward}

Our Cosserat medium is defined as $\mathcal{B}=\mathcal{D} \times \mathcal{M}$ where $\mathcal{M}$ and $\mathcal{D}$ are transformed through different kinematics. This means that we can define two kinematically independent push-foward/pull-back processes, one related to the rigid transformations of $\mathcal{M}$, the second related to the deformations of $\mathcal{D}$. We now present these two processes and refer the reader to figures 1 and 2 which provide a partial illustration of the context.

Pull-back and push-forward by the rigid transformations of $\mathcal{M}$ : Due to the presence of the microstructure in their basic constitutive definition, Cosserat media inherit from the geometric picture of the rigid body [31, in which orthonormal frames play a crucial role. In particular, any vector field $\left(\Phi_{t} \circ e\right)(\bar{X}) \mapsto$ $v(\bar{X})$ of $T\left(\Phi_{t} \circ e\right)(\mathcal{D})$ can be interpreted as a vector field of $\mathcal{E}$, i.e. a "spatial vector field" expressed in the field of mobile orthornormed frame according to $v=V^{i} t_{i}$ or pulled-back in the unique material frame of $\mathcal{B}$ through $V=R^{T} . v=V^{i} E_{i}$. Remarkably, the components of a spatial vector in its mobile frame are those of its pull-back, named the "material vector", in the material frame. Due to the orthogonality of $R$, the same relations apply to co-vector fields of $T^{*}\left(\Phi_{t} \circ e(\mathcal{D})\right)$ and finally to any Euclidean tensor field tangent to $\left(\Phi_{t} \circ e\right)(\mathcal{D})$. For the purpose of illustration (see also figure 2), let us consider the case of shells for which $p=2$, and consider the field of frames and co-frames defined by $\left(h_{1}, h_{2}\right)(\bar{X})$ and $\left(h^{1}, h^{2}\right)(\bar{X})$. They can be transformed into $\bar{X} \mapsto\left(\Gamma_{1}, \Gamma_{2}\right)(\bar{X})=\left(R^{T} . h_{1}\right.$, $\left.R^{T} . h_{2}\right)(\bar{X})$ and $\bar{X} \mapsto\left(\Gamma^{1}, \Gamma^{2}\right)(\bar{X})=\left(R^{T} \cdot h^{1}, R^{T} \cdot h^{2}\right)(\bar{X})$, which define midsurface convected frame and co-frame fields respectively, pulled back into the material frame. Note that the change of space is identical for the two fields because $R^{-1}=R^{T}$, while duality imposes $\Gamma_{\alpha} \cdot \Gamma^{\beta}=\delta_{\alpha}^{\beta}$.

Pull-back and push-forward by deformations of $\mathcal{D}$ : Due to the presence of the classical medium $\mathcal{D}$ in the definition of the Cosserat medium $\mathcal{D} \times \mathcal{M}$, a second pull-back/push-forward process holds between $T(e(\mathcal{D}))$ and $T\left(\Phi_{t} \circ e\right)(\mathcal{D})$. Using the transformation $\Phi_{t}$, any tensor field on $e(\mathcal{D})$ can be pushed forward onto $\left(\Phi_{t} \circ e\right)(\mathcal{D})$, and reciprocally pulled back from $\left(\Phi_{t} \circ e\right)(\mathcal{D})$ to $e(\mathcal{D})$ by using the restricted linear tangent maps $\nabla \bar{\Phi}_{t}=h_{\alpha} \otimes E^{\alpha}, \nabla \bar{\Phi}_{t}^{-1}=E_{\alpha} \otimes h^{\alpha}, \nabla \bar{\Phi}_{t}^{T}=E^{\alpha} \otimes h_{\alpha}$ and $\nabla \bar{\Phi}_{t}^{-T}=h^{\alpha} \otimes E_{\alpha}$. In particular, the frames and co-frames convected by the transformation are related to $\left\{E_{\alpha}\right\}_{\alpha=1, . . p}$ and $\left\{E^{\alpha}\right\}_{\alpha=1, . . p}$ through:

$$
h_{\alpha}=\nabla \bar{\Phi}_{t} \cdot E_{\alpha}, E^{\alpha}=\nabla \bar{\Phi}_{t}^{T} \cdot h^{\alpha}
$$




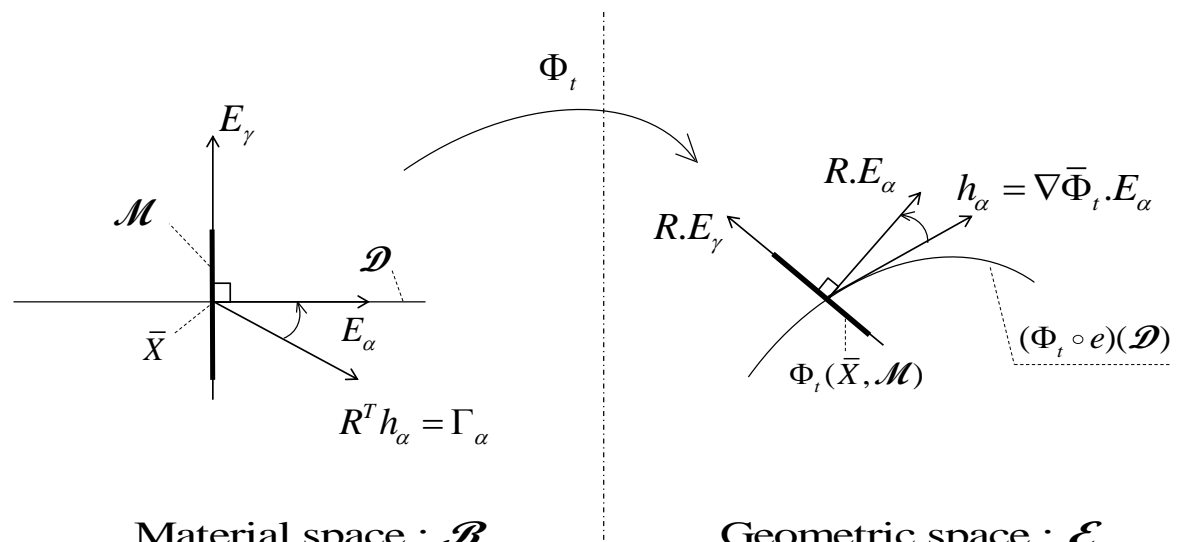

Fig. 2. Geometric picture of frames and their push-forward/pull-back relations.

However, some geometric tangent objects as the "exterior forms" and "multivectors" also involve $\sqrt{|h|}$ in this pull-back/push-forward process. This is particularly the case when considering the oriented material volume element $d X^{1} \wedge$ $d X^{2} \wedge \ldots d X^{p}$ of $\mathcal{D}$, which is changed into the metric deformed volume of same orientation $\sqrt{|h|} d X^{1} \wedge d X^{2} \wedge \ldots d X^{p}$ by the transformation $\Phi_{t} \circ e$. Similarly, the oriented material area element in any point $\bar{X}$ of $\mathcal{D}$ is defined as the $p-1$ form $d Y^{1} \wedge d Y^{2} \ldots \wedge d Y^{p-1}$, where $\left\{Y^{\gamma}\right\}_{\gamma=1,2 \ldots p-1}$ is a Cartesian chart, of natural orthonormal basis $\left\{\bar{H}_{\gamma}=\partial / \partial Y^{\gamma}\right\}_{\gamma=1,2 . . p-1}$, covering a material hyperplane crossing $\bar{X}$. This form is transformed into $|\bar{h}|^{1 / 2} d Y^{1} \wedge d Y^{2} \wedge \ldots d Y^{p-1}$ by the deformation, with $|\bar{h}|$ the determinant of the fundamental tensor of $\mathcal{E}$ in the convected basis $\left\{\bar{h}_{\gamma}=\nabla \bar{\Phi}_{t} \cdot \bar{H}_{\gamma}=\partial r / \partial Y^{\gamma}\right\}_{\gamma=1,2 . . p-1}$. Exploiting duality of $(p-1)$-forms and $(p-1)$-vectors, we may define the material oriented area element as the $(p-1)$-vector $\bar{H}_{1} \wedge \bar{H}_{2} \ldots \wedge \bar{H}_{p-1}$, which can be represented by the conjugate true vector of $T^{*} e(\bar{X})[32]$ :

$$
\nu=\nu_{\alpha} E^{\alpha}=\frac{1}{(p-1) !} \epsilon_{\alpha \alpha_{1} \ldots \alpha_{p-2} \alpha_{p-1}}\left|\frac{\partial\left(X^{\alpha_{1}}, X^{\alpha_{2}} \ldots, X^{\alpha_{p-1}}\right)}{\partial\left(Y^{1}, Y^{2}, Y^{3} \ldots, Y^{p-1}\right)}\right| E^{\alpha}
$$

which defines the unit normal to the element, where $\left|\frac{\partial(,, \ldots,)}{\partial(,, \ldots,)}\right|$ denotes a functional determinant, while for any integer $n, \epsilon_{i_{1} i_{2} \ldots i_{n}}$ is equal to zero if two indices 
are identical, equal to +1 (respectively -1 ), if $\left(i_{1}, i_{2}, \ldots i_{n}\right)$ is an even (respect. odd) permutation of $(1,2, \ldots n)$. Similarly, normalizing the conjugate vector of $\bar{h}_{1} \wedge \bar{h}_{2} \ldots \wedge \bar{h}_{p-1}$, defines the unit normal to the oriented deformed element as the covector:

$$
\nu_{t}=\nu_{t, \alpha} h^{\alpha}=\left|\frac{h}{\bar{h}}\right|^{1 / 2} \frac{1}{(p-1) !} \epsilon_{\alpha \alpha_{1} \ldots \alpha_{p-2} \alpha_{p-1}}\left|\frac{\partial\left(X^{\alpha_{1}}, X^{\alpha_{2}} \ldots, X^{\alpha_{p-1}}\right)}{\partial\left(Y^{1}, Y^{2}, Y^{3} \ldots, Y^{p-1}\right)}\right| h^{\alpha} .
$$

Note that the two vectors $\nu$ and $\nu_{t}$ whose components are related by $\nu_{t, \alpha}=$ $(|h| /|\bar{h}|)^{1 / 2} \nu_{\alpha}$, contain all the information about the orientation of the original $(p-1)$-forms. Moreover, defining the measure (area) of the material and deformed elements as $d S=d Y^{1} \ldots d Y^{p-1}$ and $d S_{t}=|\bar{h}|^{1 / 2} d Y^{1} \ldots d Y^{p-1}$ respectively, where each $d Y^{\gamma}$ represents the components of an infinitesimal vector $d Y^{\gamma} \bar{H}_{\gamma}$ (with no summation on $\gamma=1,2 \ldots p-1$ ), we also have the relation between the co-vectors $\nu_{t} d S_{t}$ and $\nu d S$ which will be used in the subsequent developments instead of the original $(p-1)$ forms:

$$
\nu_{t} d S_{t}=\left(\nu_{t, \alpha} d S_{t}\right) h^{\alpha}=\left(\sqrt{|h|} \nu_{\alpha} d S\right) \nabla \bar{\Phi}_{t}^{-T} \cdot E^{\alpha}=\sqrt{|h|} \nabla \bar{\Phi}_{t}^{-T} \cdot(\nu d S) .
$$

Taking $d S=|\bar{H}|^{1 / 2} d Y^{1} \ldots d Y^{p-1}$, (12) holds for a relation between material and deformed oriented area elements of any hyper-surface of $\mathcal{D}$ parameterically defined by $X^{\alpha}\left(Y^{\gamma}\right)$, with $|\bar{H}|$ the determinant of the metric of $\mathcal{D}$ in the natural basis $\left\{\bar{H}_{\gamma}\right\}_{\gamma=1 . . p-1}$ covering the hyper-surface.

\subsection{Lagrangian of a Cosserat medium}

On the definition (3) of the configuration space, the Lagrangian of a Cosserat medium $\mathcal{B}=\mathcal{D} \times \mathcal{M}$ at current time $t$ is defined as the following functional:

$$
L=\int_{\mathcal{D}} \mathcal{L}_{t}\left(\mathrm{~g}, \frac{\partial \mathrm{g}}{\partial t}, \frac{\partial \mathrm{g}}{\partial X^{\alpha}}\right) \sqrt{|h|} d X^{1} \wedge d X^{2} \wedge \ldots \wedge d X^{p},
$$

where $\mathcal{L}_{t} \sqrt{|h|} d X^{1} \wedge \ldots \wedge d X^{p}$ is the Lagrangian volume-form of the Cosserat medium, and $\mathcal{L}_{t}$ is the density of Lagrangian per unit of metric volume $\sqrt{|h|} d X^{1} \wedge$ $d X^{2} \wedge \ldots \wedge d X^{p}$ of the current deformed configuration $\left(\Phi_{t} \circ e\right)(\mathcal{D})$. Alternatively, $L$ can be related to the volume of reference configuration $\left(\Phi_{o} \circ e\right)(\mathcal{D})$ as:

$$
L=\int_{\mathcal{D}} \mathcal{L}_{o}\left(\mathrm{~g}, \frac{\partial \mathrm{g}}{\partial t}, \frac{\partial \mathrm{g}}{\partial X^{\alpha}}\right) \sqrt{\left|h_{o}\right|} d X^{1} \wedge d X^{2} \wedge \ldots \wedge d X^{p}
$$

or directly to the volume of parametric (material) space $\mathcal{D}$ :

$$
L=\int_{\mathcal{D}} \mathcal{L}\left(\mathrm{g}, \frac{\partial \mathrm{g}}{\partial t}, \frac{\partial \mathrm{g}}{\partial X^{\alpha}}\right) d X^{1} \wedge d X^{2} \wedge \ldots \wedge d X^{p}
$$

In (14) (respectively (15) $\mathcal{L}_{o}$, (respect. $\mathcal{L}$ ), is the density of the Lagrangian of $\mathcal{B}$ per unit of metric volume $\sqrt{\left|h_{o}\right|} d X^{1} \wedge d X^{2} \wedge \ldots \wedge d X^{p}$ (respect. non-metric volume $\left.d X^{1} \wedge d X^{2} \wedge \ldots \wedge d X^{p}\right)$ of $\left(\Phi_{o} \circ e\right)(\mathcal{D})$ (respect., of $\left.\mathcal{D}\right)$. 


\subsection{Reduction of the Lagrangian}

Physically, the Lagrangian $L$ depends on $\partial \mathrm{g} / \partial t$ through the kinetic energy of $\mathcal{B}$, and on $\partial \mathrm{g} / \partial X^{\alpha}$ through the internal strain energy of its material that is assumed to be hyperelastic. The three Lagrangian densities $\mathcal{L}, \mathcal{L}_{o}$ and $\mathcal{L}_{t}$ are left invariant in the sense that substituting $\mathrm{g}$ by $\mathrm{kg}$, in any of them (with $\mathrm{k}$ a constant transformation in $G$ over each point of space-time $\mathcal{D} \times \mathbb{R}^{+}$), does not change its value. Physically, this reflects the fact that both the densities of kinetic and internal strain energy are the same when observed from any frame of geometric space. The first property is a key result of rigid body mechanics [31], while the second is a consequence of the material frame indifference of mechanics of continuous media [33]. As a result, taking $\mathrm{k}=\mathrm{g}^{-1}$, allows the transformation of the above three Lagrangian into a unique reduced Lagrangian, which can be written, if, for instance, we start from (13), as:

$$
L_{r}=\int_{\mathcal{D}} \mathfrak{L}_{t}\left(\eta, \xi_{\alpha}\right) \sqrt{|h|} d X^{1} \wedge d X^{2} \wedge \ldots \wedge d X^{p}
$$

where we introduced the following left invariant vector fields leaving in the Lie algebra $\mathfrak{g}$ of $G$ (considered as a group of matrices):

$$
\eta=\mathrm{g}^{-1} \frac{\partial \mathrm{g}}{\partial t}, \xi_{\alpha}=\mathrm{g}^{-1} \frac{\partial \mathrm{g}}{\partial X^{\alpha}}, \alpha=1,2 \ldots p
$$

In (16), $\mathfrak{L}_{t}$ defines the reduced Lagrangian volume density per unit of metric volume of deformed configuration $\left(\Phi_{t} \circ e\right)(\mathcal{D})$, while $|h|$ is by definition, the determinant of the matrix $h_{\alpha \beta}=h_{\alpha} \cdot h_{\beta}=\left(R^{T} .\left(\partial r / \partial X^{\alpha}\right)\right) \cdot\left(R^{T} .\left(\partial r / \partial X^{\beta}\right)\right)=\Gamma_{\alpha} \cdot \Gamma_{\beta}$. Then, using (5) in (17), shows that $\Gamma_{\alpha}$ is the linear (opposed to angular) component of $\xi_{\alpha}$. Thus $\sqrt{|h|}$ only depends on the left invariant fields $\xi_{\alpha}$, and (16) does define, as a whole, a reduced Lagrangian $L_{r}$ in the Lie algebra $\mathfrak{g}$.

Alternatively, applying the same reduction process to (14) or (15) instead of (13), permits the reduced Lagrangian densities to be defined per unit of metric volume of reference configuration $\left(\Phi_{o} \circ e\right)(\mathcal{D})$ and per unit of non-metric (material) volume $\mathcal{D}$, denoted $\mathfrak{L}_{o}$ and $\mathfrak{L}$ respectively. As $\mathfrak{L}_{t}$, these two further densities only depend on $\left(\eta, \xi_{\alpha}\right)$. Moreover, in each of these two other cases, the volume element does not depend on the current configuration and the corresponding Lagrangian is left invariant too. Finally equating the reduced versions of (13), (14) and (15), allows stating the following relations between the three reduced Lagrangian densities:

$$
\mathfrak{L}=\mathfrak{L}_{o} \sqrt{\left|h_{o}\right|}=\mathfrak{L}_{t} \sqrt{|h|},
$$

In the following, we start from the density $\mathfrak{L}$ related to the material space and will introduce later the case of densities $\mathfrak{L}_{o}$ and $\mathfrak{L}_{t}$. 


\section{Poincaré's equations of Cosserat media in the material space}

In this section we quickly remind the construction of [1] leading to the balance equations of multi-dimensional Cosserat in the material space, i.e., in the space of the labels of the micro-structures. These equations will be a first step toward their extension to the geometric space, namely in the reference and deformed configuration, in which they will take the consistent form required by their practical use. Following Poincaré's approach [2], a Cosserat medium $\mathcal{B}=\mathcal{D} \times \mathcal{M}$ subjected to a set of external forces is governed by the extended Hamilton principle, which can be stated directly on the definition $(3)$ of $\mathcal{C}$ ( $\alpha$ running from 1 to $p$ ), as:

$$
\delta \int_{t_{1}}^{t_{2}} \int_{\mathcal{D}} \mathfrak{L}\left(\eta, \xi_{\alpha}\right) d X^{1} \wedge d X^{2} \wedge \ldots \wedge d X^{p} d t=-\int_{t_{1}}^{t_{2}} \delta W_{\text {ext }} d t,
$$

for any $\delta \mathrm{g}=\mathrm{g} \delta \zeta$ where $\delta \zeta \in \mathfrak{g}$ is a field of material variation of $\mathrm{g}$ achieved while $t$ and all the $X^{\alpha}$ are kept fixed. In $(19), \delta W_{\text {ext }}$ models the virtual work of external forces and can be detailed as:

$$
\begin{gathered}
\delta W_{\text {ext }}=\int_{\mathcal{D}}<F_{\text {ext }}, \delta \zeta>d X^{1} \wedge d X^{2} \wedge \ldots \wedge d X^{p}+ \\
\int_{\partial \mathcal{D}}<\bar{F}_{\text {ext }}, \delta \zeta>|\bar{H}|^{1 / 2} d Y^{1} \wedge d Y^{2} \wedge \ldots \wedge d Y^{p-1},
\end{gathered}
$$

with $<., .>$ the duality product in the Lie algebra $\mathfrak{g},\left(Y^{\gamma}\right)_{\gamma=1,2 . . p-1}$, a set of material coordinates covering the boundary $\partial \mathcal{D}$. Finally, $F_{\text {ext }}$ and $\bar{F}_{\text {ext }}$ are densities of external forces in $\mathfrak{g}^{*}$ per unit of volume of $\mathcal{D}$ and $\partial \mathcal{D}$ respectively. Note that we consider boundaries subjected to external forces only, the case of imposed motions being easily modelled by defining two types of boundaries. Also note that for beams, i.e. one-dimensional Cosserat media, one has to take:

$$
|\bar{H}|^{1 / 2}=\delta_{\text {irac }}(\partial \mathcal{D}),
$$

where $\delta_{\text {irac }}$ is the Dirac distribution, and 20 has to be integrated within the meaning of distributions. Now, let us invoke the constraints of variation at fixed time and material labels:

$$
\delta \frac{\partial \mathrm{g}}{\partial t}=\frac{\partial \delta \mathrm{g}}{\partial t}, \delta \frac{\partial \mathrm{g}}{\partial X_{\alpha}}=\frac{\partial \delta \mathrm{g}}{\partial X_{\alpha}}, \text { for } \alpha=1,2 . . p .
$$

Then inserting $" \delta \mathrm{g}=\mathrm{g} \delta \zeta$ " into (22) gives the following relations, as first derived by Poincaré [2, which play a key role in the variational calculus on Lie groups [34]:

$$
\delta \eta=\frac{\partial \delta \zeta}{\partial t}+a d_{\eta}(\delta \zeta), \delta \xi_{\alpha}=\frac{\partial \delta \zeta}{\partial X^{\alpha}}+a d_{\xi_{\alpha}}(\delta \zeta),
$$

with $a d$ the adjoint map of $\mathfrak{g}$ on itself. As detailed in [11, applying the standard variational calculus to $(19)$ with $(23)$ running before the usual by part integration 
in time, and the divergence theorem, gives the Poincaré equations of a Cosserat medium in the material frame (we use summation convention on repeated indices $\alpha)$ :

$$
\begin{aligned}
& \frac{\partial}{\partial t}\left(\frac{\partial \mathfrak{L}}{\partial \eta}\right)-a d_{\eta}^{*}\left(\frac{\partial \mathfrak{L}}{\partial \eta}\right)+\frac{\partial}{\partial X^{\alpha}}\left(\frac{\partial \mathfrak{L}}{\partial \xi_{\alpha}}\right)-a d_{\xi_{\alpha}}^{*}\left(\frac{\partial \mathfrak{L}}{\partial \xi_{\alpha}}\right)=F_{e x t}, \\
& \frac{\partial \mathfrak{L}}{\partial \xi_{\alpha}} \nu_{\alpha}=-\bar{F}_{e x t}
\end{aligned}
$$

where $a d^{*}$ is the co-adjoint map of $\mathfrak{g}$ on $\mathfrak{g}^{*}$, while $\nu=\nu_{\alpha} E^{\alpha}$ is the unit outward normal to $e(\mathcal{D})$, and $\partial \mathfrak{L} / \partial \eta$, and $\partial \mathfrak{L} / \partial \xi_{\alpha}$ define some conjugate generalized moments that will be detailed in the following.

These equations represent the dynamics of a Cosserat medium reduced in the dual of its Lie algebra here identified as the space of infinitesimal material (right) transformations of $G$. They govern the time-evolution of the material velocities $\eta$ and when the external forces are left invariant, they can be time-integrated separately to compute the velocity field in a first step. In a second step, the motion of the medium can be reconstructed by using the so-called reconstruction equation, which can be simply stated as:

$$
\forall \bar{X} \in \mathcal{D}: \frac{\partial \mathrm{g}}{\partial t}(\bar{X}, t)=(\mathrm{g} \eta)(\bar{X}, t) .
$$

In all cases (symmetric or not), (25) supplements (24), to give a set of timeevolution equations in the following definition of a Cosserat medium's state space:

$$
\mathcal{S}=\{(\mathrm{g}, \eta): \bar{X} \in \mathcal{D} \mapsto(\mathrm{g}, \eta)(\bar{X}) \in G \times \mathfrak{g}\}
$$

Remark 3.1: Unfortunately, these equations are not directly exploitable in practise since they are stated in the material space of microstructure labels $\mathcal{D}$, i.e., in a non metric space a priori disconnected from the geometric (physical) space. The purpose of what follows is to give them the physical consistency required by their application. This will be done by reexpressing the medium dynamics in terms of the metric densities $\mathfrak{L}_{o}$ and $\mathfrak{L}_{t}$. At the end, we will obtain two new sets of equations holding for the Poincaré equations of Cosserat media in the reference and deformed configuration $\left(\Phi_{o} \circ e\right)(\mathcal{D})$ and $\left(\Phi_{t} \circ e\right)(\mathcal{D})$ respectively. These further equations stated in section 5 will allow recovering several geometrically exact formulations for shells in sections $6,7,8$. To derive them, we need to enter further into the geometric model underlaid by $(24,25)$.

\section{Geometric model of Cosserat media}

The variational calculus leading to (24) handles velocity-type vectors $\left(\eta, \xi_{\alpha}\right)$ and force-type vectors $\left(\partial \mathfrak{L} / \partial \eta, \partial \mathfrak{L} / \partial \xi_{\alpha}, F_{\text {ext }}, \bar{F}_{\text {ext }}\right)$ of $\mathfrak{g}$ and $\mathfrak{g}^{*}$ respectively, which are dual of each other through the duality product $<., .>$. However, this 
calculus hides a further dimensionality involving the space-time base-manifold $\mathcal{D} \times \mathbb{R}^{+}[8]$. Though not readily apparent, this further aspect of the theory strongly structures the geometric model of Cosserat-media and especially that of internal stress. The purpose of this section is to introduce this aspect and to use it to prepare the ground for the model of Cosserat shells as it will be discussed in the further sections. To introduce this important point, we will first build on the concept of duality.

\subsection{Duality in the material space}

We consider in this section a material Lagrangian density $\mathfrak{L}$ in the form $\mathfrak{L}\left(\eta, \xi_{\alpha}\right)=$ $\mathfrak{T}(\eta)-\mathfrak{U}\left(\xi_{\alpha}\right)$, with $\mathfrak{T}$ and $\mathfrak{U}$, the density of kinetic and internal elastic potential energy per unit of material volume respectively. Let us first remark that in the Poincaré-Cosserat picture, $\eta$ and $\xi_{\alpha=1,2 \ldots p}$ are not only vector fields in the Lie algebra but also the components of a unique field of 1-form on space-time, with value in the Lie algebra $\mathfrak{g}$ of $G\left[11\right.$. Endowing $\mathfrak{g}$ with a basis $\left\{l_{1}, l_{2} \ldots l_{n}\right\}$, such a field, here generically noted as $\Upsilon$, is defined as $\Upsilon:(\bar{X}, t) \in \mathcal{D} \times \mathbb{R}^{+} \mapsto \Upsilon(\bar{X}, t) \in$ $\mathfrak{g} \otimes \wedge T^{*}\left(\mathcal{D} \times \mathbb{R}^{+}\right)$, and may be detailed as:

$$
\Upsilon(\bar{X}, t)=\Upsilon_{0}^{j} l_{j} \otimes d t+\Upsilon_{\beta}^{j} l_{j} \otimes d X^{\beta},
$$

where 0 denotes the coordinate-index along time axis, i.e., $X^{0}=t$, while in the case of the left-invariant fields of $(17), \Upsilon_{0}^{j} l_{j}=\eta$ and $\Upsilon_{\beta}^{j} l_{j}=\xi_{\beta}$. Similarly, the

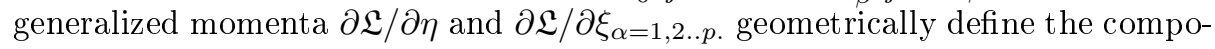
nents of a field in the dual of the space of $\Upsilon$, i.e. a unique vector field on spacetime with components in the dual of the Lie algebra of $G$ of basis $\left\{\omega^{1}, \omega^{2}, \ldots \omega^{n}\right\}$. Such a field, generically defined as $\Lambda:(\bar{X}, t) \in \mathcal{D} \times \mathbb{R}^{+} \mapsto \mathfrak{g}^{*} \otimes T\left(\mathcal{D} \times \mathbb{R}^{+}\right)$is detailed as:

$$
\Lambda(\bar{X}, t)=\Lambda_{i}^{0} \omega^{i} \otimes \partial / \partial t+\Lambda_{i}^{\alpha} \omega^{i} \otimes \partial / \partial X^{\alpha},
$$

where in the case of our generalized momenta, we have $\Lambda_{i}^{0} \omega^{i}=\partial \mathfrak{L} / \partial \eta=\partial \mathfrak{T} / \partial \eta$ and $\Lambda_{i}^{\alpha} \omega^{i}=-\partial \mathfrak{L} / \partial \xi_{\alpha}=\partial \mathfrak{U} / \partial \xi_{\alpha}$. With these definitions, in each point of spacetime, any $\Lambda$ linearly acts on any $\Upsilon$ according to the following (double) duality product denoted $(<., .>)$ :

$$
(<\Lambda, \Upsilon>)=\Lambda_{i}^{0} \Upsilon_{0}^{j}<\omega^{i}, l_{j}>(\partial / \partial t, d t)+\Lambda_{i}^{\alpha} \Upsilon_{\beta}^{j}<\omega^{i}, l_{j}>\left(\partial / \partial X^{\alpha}, d X^{\beta}\right),
$$

with $\left\langle\omega^{i}, l_{j}\right\rangle=\delta_{j}^{i}$, while $(.,$.$) is another duality product requiring considering$ vectors $v$ of $T\left(\mathcal{D} \times \mathbb{R}^{+}\right)$as linear functional acting on 1-forms $\omega$ of $T^{*}\left(\mathcal{D} \times \mathbb{R}^{+}\right)$ according to $v(\omega)=\omega(v)=(\omega, v)=(v, \omega)$. With these considerations, and since $\left(\partial . / \partial X^{i}, d X^{j}\right)=\delta_{i}^{j}\left(\right.$ with $\left.X^{0}=t\right)$, we simply have:

$$
(<\Lambda, \Upsilon>)=\Lambda_{i}^{0} \Upsilon_{0}^{i}+\Lambda_{i}^{\alpha} \Upsilon_{\alpha}^{i}=<\Lambda^{0}, \Upsilon_{0}>+<\Lambda^{\alpha}, \Upsilon_{\alpha}>=\left(\Lambda_{i}, \Upsilon^{i}\right),
$$

which appears at the very beginning of the above variational calculus in the virtual work of the inertial and internal forces and in all its consequences. In the subsequent developments, the vectors $\Upsilon_{0}=\Upsilon_{0}^{i} l_{i}$ and $\Upsilon_{\alpha}=\Upsilon_{\alpha}^{i} l_{i}$ are velocity-type 
vectors, or in rigid body mechanics' terminology, are "twists" of $\mathfrak{g}$. On the dual side, $\Lambda^{0}=\Lambda_{i}^{0} \omega^{i}$ and $\Lambda^{\alpha}=\Lambda_{i}^{\alpha} \omega^{i}$ are force-type vectors or "wrenches" of $\mathfrak{g}^{*}$. Moreover, from the above context, the components of the two fields (27) and 28) in their respective basis of $\mathfrak{g}$ and $\mathfrak{g}^{*}$, can be written as: $\Upsilon^{i}=\Upsilon_{0}^{i} d t+\Upsilon_{\alpha}^{2} d X^{\alpha}$ and $\Lambda_{i}=\Lambda_{i}^{0} \partial / \partial t+\Lambda_{i}^{\alpha} \partial / \partial X^{\alpha}$ which define 1-forms acting on $T\left(\mathcal{D} \times \mathbb{R}^{+}\right)$and $T^{*}\left(\mathcal{D} \times \mathbb{R}^{+}\right)$respectively. Kinematically, the $\Upsilon^{i}$ twist components model the space-time variations of the rigid microstructure $\mathcal{M}$-configuration (in $G$ ) in any point of $\mathcal{D} \times \mathbb{R}^{+}$when shifting along any direction of $\mathcal{D} \times \mathbb{R}^{+}$, while the $\Lambda_{i}$ wrench components model the kinetic momentum of each copy of $\mathcal{M}$ above $\mathcal{D}$ and the stress exerted on it. We will detail further these relationships in section 4.5 after shifting this context to the reference and deformed configuration in subsection 4.2 and introducing the role of densities and volume forms in subsection 4.3.

\subsection{Duality in the reference and deformed configuration}

The above context can be shifted to the reference and deformed configuration by using the push-forward and pull-back operations in the convected frame and co-frame fields tangent to $\left(\Phi_{o} \circ e\right)(\mathcal{D})$ and $\left(\Phi_{t} \circ e\right)(\mathcal{D})$ introduced in section 2.3 and 2.4. In this context, the field $\Upsilon=\Upsilon_{0} d t+\Upsilon_{\beta} d X^{\beta}$ transforms into:

$$
\begin{aligned}
& \Upsilon_{o}=\Upsilon_{0} d t+\Upsilon_{\beta} h_{o}^{\beta}, \\
& \Upsilon_{t}=\Upsilon_{0} d t+\Upsilon_{\beta} h^{\beta},
\end{aligned}
$$

on the reference configuration (30a), and the deformed (30b). On the dual side, the field $\Lambda=\Lambda^{0} \partial / \partial t+\Lambda_{o}^{\alpha} \partial / \partial X^{\alpha}$ transforms into:

$$
\begin{aligned}
& \Lambda_{o}=\Lambda^{0} \partial / \partial t+\Lambda_{o}^{\alpha} h_{o, \alpha}, \\
& \Lambda_{t}=\Lambda^{0} \partial / \partial t+\Lambda_{t}^{\alpha} h_{\alpha},
\end{aligned}
$$

on the reference and deformed configuration. Note that in (31), the transformation does not only affect the basis vectors of $\Lambda$ (by pull-back) but also its components, since as we will see in the next section, $\Lambda$ is not a true tensor but a volume density (and its transformation involves $\sqrt{|h|}$ as mentioned in section 2.4). With these further convected fields, the duality product (29) still holds, but between the convected frame and co-frame fields $\left(h_{\alpha}\right)_{\alpha=1,2 . . p}$ and $\left(h^{\alpha}\right)_{\alpha=1,2 \ldots p}$, and we have:

$$
(<\Lambda, \Upsilon>)=\left(<\Lambda_{o}, \Upsilon_{o}>\right)=\left(<\Lambda_{t}, \Upsilon_{t}>\right) .
$$

Following the remark at the end of the previous sub-section, $\Upsilon_{o}$ and $\Upsilon_{t}$ still model the space-time variations of the microstructure configuration (in $G$ ), but when shifting along the metric coordinates lines drawn on the reference and deformed configurations by the convection of the material chart. Similarly, $\Lambda_{o}$ and $\Lambda_{t}$ model the kinetic momentum of the micro-structures and the stress exerted on them, but related to the reference and deformed configuration in a way we will

detail in the next section after introducing the role of densities and volume forms in the next subsection. 


\subsection{Volume forms and densities}

All the terms of the equilibrium described in (24) define densities of wrench in $\mathfrak{g}^{*}$ related to the metric volume of $\mathcal{D}(24 \mathrm{a})$, and $\partial \mathcal{D}(24 \mathrm{~b})$. In particular, the external volume forces involved in (20), are intrinsically defined as the volumeform field on $\mathcal{D}$ with values in $\mathfrak{g}^{*}: F_{\text {ext }} d X^{1} \wedge d X^{2} \wedge \ldots \wedge d X^{p}$ which transforms into:

$$
F_{e x t, o} \sqrt{\left|h_{o}\right|} d X^{1} \wedge d X^{2} \wedge \ldots \wedge d X^{p}, F_{e x t, t} \sqrt{|h|} d X^{1} \wedge d X^{2} \wedge \ldots \wedge d X^{p},
$$

in the reference and current deformed configuration. In the same way, the external boundary forces (exerted on $\partial \mathcal{D}$ ) are defined by volume-form fields on the boundaries of $\mathcal{D}$ with value in $\mathfrak{g}^{*}$ of the type $\bar{F}_{\text {ext }} d Y^{1} \wedge d Y^{2} \wedge \ldots \wedge d Y^{p-1}$, which transform into:

$$
\bar{F}_{e x t, o}\left|\overline{h_{o}}\right|^{1 / 2} d Y^{1} \wedge d Y^{2} \wedge \ldots \wedge d Y^{p-1}, \bar{F}_{e x t, t}|\bar{h}|^{1 / 2} d Y^{1} \wedge d Y^{2} \wedge \ldots \wedge d Y^{p-1} .
$$

on the reference and deformed configuration. Similarly, any space-time material conjugate momentum of the type $\Lambda$ defined in $(28)$ is in fact the unique component of a volume-form field $\Lambda \otimes d X^{1} \wedge d X^{2} \wedge \ldots \wedge d X^{p}=\left(\Lambda^{0} \partial / \partial t+\Lambda^{\alpha} E_{\alpha}\right) \otimes$ $d X^{1} \wedge d X^{2} \wedge \ldots \wedge d X^{p}$, with values in $\mathfrak{g}^{*} \otimes T\left(\mathcal{D} \times \mathbb{R}^{+}\right)$which transforms into:

$$
\begin{gathered}
\Lambda_{o} \sqrt{\left|h_{o}\right|} \otimes d X^{1} \wedge d X^{2} \wedge \ldots \wedge d X^{p}= \\
\left(\Lambda_{o}^{0} \sqrt{\left|h_{o}\right|} \partial / \partial t+\Lambda_{o}^{\alpha} \sqrt{\left|h_{o}\right|} h_{o \alpha}\right) \otimes d X^{1} \wedge d X^{2} \wedge \ldots \wedge d X^{p}
\end{gathered}
$$

on the reference configuration, and into:

$$
\begin{gathered}
\Lambda_{t} \sqrt{|h|} \otimes d X^{1} \wedge d X^{2} \wedge \ldots \wedge d X^{p}= \\
\left(\Lambda_{t}^{0} \sqrt{|h|} \partial / \partial t+\Lambda_{t}^{\alpha} \sqrt{|h|} h_{\alpha}\right) \otimes d X^{1} \wedge d X^{2} \wedge \ldots \wedge d X^{p},
\end{gathered}
$$

on the deformed configuration. The three volume densities $\Lambda, \Lambda_{o}$ and $\Lambda_{t}$ are related to the material volume element, the metric reference and the metric de-

formed volume element respectively, and are apparent in the following functional duality product between fields:

$$
\int_{\mathcal{D}}(<\Lambda, \Upsilon>) d \mathcal{D}=\int_{\mathcal{D}}\left(<\Lambda_{o}, \Upsilon_{o}>\right) \sqrt{\left|h_{o}\right|} d \mathcal{D}=\int_{\mathcal{D}}\left(<\Lambda_{t}, \Upsilon_{t}>\right) \sqrt{|h|} d \mathcal{D},
$$

which completes the intrinsic model of conjugate momenta of (24).

\section{Poincaré's equations of Cosserat media in the reference and deformed configuration}

Based on the above geometric model, this section aims at deducing from the Poincare equations in the material space (24), two other sets of equations enjoying more physical insights: one related to the reference configuration, the other 
related to the deformed configuration. To derive these two further sets of equations, let us first remark that from the previous section, the external forces (33) and the space-time generalized momenta (35), and (36) are volume-densities which behave like the Lagrangian densities $\mathfrak{L}, \mathfrak{L}_{o}$ and $\mathfrak{L}_{t}$, i.e. obey the following relations, similar to (18), when shifting from the material to the reference and current configuration:

$$
\begin{aligned}
& F_{\text {ext }}=F_{\text {ext }, o} \sqrt{\left|h_{o}\right|}=F_{\text {ext }, t} \sqrt{|h|}, \\
& \Lambda=\sqrt{\left|h_{o}\right|} \Lambda_{o}=\sqrt{|h|} \Lambda_{t} .
\end{aligned}
$$

In the same way, the external surface forces (34) define volume-densities on the boundaries of $\mathcal{D}$ which behave as:

$$
\bar{F}_{e x t}|\bar{H}|^{1 / 2}=\bar{F}_{e x t, o}\left|\bar{h}_{o}\right|^{1 / 2}=\bar{F}_{e x t, t}|\bar{h}|^{1 / 2}
$$

In $(38)$ and 39$),\left(F_{\text {ext }, o}, \bar{F}_{\text {ext,o }}\right)$ and $\left(F_{\text {ext }, t}, \bar{F}_{\text {ext }, t}\right)$ denote the densities of the external wrenches exerted on $\mathcal{B}$, per unit of metric-volume of $\left(\left(\Phi_{o} \circ e\right)(\mathcal{D}), \partial\left(\Phi_{o} \circ\right.\right.$ $e)(\partial \mathcal{D}))$, and per unit of metric volume of $\left(\left(\Phi_{t} \circ e\right)(\mathcal{D}), \partial\left(\Phi_{t} \circ e\right)(\partial \mathcal{D})\right)$ respectively. Similarly, as this will be detailed in the next section, the $\Lambda_{o}^{\alpha}$ and $\Lambda_{t}^{\alpha}$ (respectively $\Lambda_{o}^{0}$ and $\Lambda_{t}^{0}$ ) define some densities of internal stress wrench (respectively the densities of kinetic momentum wrench) per unit of metric volume of $\left(\Phi_{o} \circ e\right)(\mathcal{D})$ and of metric volume of $\left(\Phi_{t} \circ e\right)(\mathcal{D})$.

For the time being, let us remark that introducing $\mathfrak{L}=\mathfrak{L}_{o} \sqrt{\left|h_{o}\right|}=\mathfrak{L}_{t} \sqrt{|h|}$ into the conjugate momentums $\partial \mathfrak{L} / \partial \eta$ and $\partial \mathfrak{L} / \partial \xi^{\alpha}$ of the material Poincaré equations (24), and using the identities (38b), allows the following relations between the densities $\Lambda, \Lambda_{o}$ and $\Lambda_{t}$ and the Lagrangian densities $\mathfrak{L}, \mathfrak{L}_{o}$ and $\mathfrak{L}_{t}$ to be stated:

$$
\begin{gathered}
\Lambda^{0}=\frac{\partial \mathfrak{L}}{\partial \eta}, \Lambda_{o}^{0}=\frac{\partial \mathfrak{L}_{o}}{\partial \eta}, \Lambda_{t}^{0}=\frac{\partial \mathfrak{L}_{t}}{\partial \eta} \\
\Lambda^{\alpha}=-\frac{\partial \mathfrak{L}}{\partial \xi_{\alpha}}, \Lambda_{o}^{\alpha}=-\frac{\partial \mathfrak{L}_{o}}{\partial \xi_{\alpha}}, \Lambda_{t}^{\alpha}=-\frac{\partial \mathfrak{L}_{t}}{\partial \xi_{\alpha}}-\frac{1}{\sqrt{|h|}} \frac{\partial \sqrt{|h|}}{\partial \xi_{\alpha}} \mathfrak{L}_{t} .
\end{gathered}
$$

where we used the fact that $\left|h_{o}\right|$ is independent of $\eta$ and the $\xi_{\alpha}$ 's, while $|h|$ depends on the $\xi_{\alpha}$ 's only. For the purpose of concision, we will write the last relation of (41), as:

$$
\Lambda_{t}^{\alpha}=-\frac{\partial \mathfrak{L}_{t}}{\partial \xi_{\alpha}}-\frac{1}{\sqrt{|h|}} \frac{\partial \sqrt{|h|}}{\partial \xi_{\alpha}} \mathfrak{L}_{t} \triangleq-\left[\frac{\partial \mathfrak{L}}{\partial \xi_{\alpha}}\right]_{t} .
$$

Now, introducing all these relations (from $(38)$ to $(42)$ ) in the original material Poincaré equations (24) allows to state: 
- The Poincaré-equations of Cosserat media in the reference configuration:

$$
\begin{aligned}
& \frac{1}{\sqrt{\left|h_{o}\right|}}\left(\frac{\partial}{\partial t}\left(\sqrt{\left|h_{o}\right|}\left(\frac{\partial \mathfrak{L}_{o}}{\partial \eta}\right)\right)-a d_{\eta}^{*}\left(\sqrt{\left|h_{o}\right|}\left(\frac{\partial \mathfrak{L}_{o}}{\partial \eta}\right)\right)\right)+ \\
& \frac{1}{\sqrt{\left|h_{o}\right|}}\left(\frac{\partial}{\partial X^{\alpha}}\left(\sqrt{\left|h_{o}\right|}\left(\frac{\partial \mathfrak{L}_{o}}{\partial \xi_{\alpha}}\right)\right)-a d_{\xi_{\alpha}}^{*}\left(\sqrt{\left|h_{o}\right|}\left(\frac{\partial \mathfrak{L}_{o}}{\partial \xi_{\alpha}}\right)\right)\right)=F_{\text {ext }, o}, \\
& \left(\frac{\left|h_{o}\right|}{\left|\bar{h}_{o}\right|}\right)^{1 / 2}\left(\frac{\partial \mathfrak{L}_{o}}{\partial \xi_{\alpha}}\right) \nu_{\alpha}=-\bar{F}_{\text {ext }, o},
\end{aligned}
$$

- The Poincaré-equations of Cosserat media in the deformed configuration:

$$
\begin{aligned}
& \frac{1}{\sqrt{|h|}}\left(\frac{\partial}{\partial t}\left(\sqrt{|h|}\left(\frac{\partial \mathfrak{L}_{t}}{\partial \eta}\right)\right)-a d_{\eta}^{*}\left(\sqrt{|h|}\left(\frac{\partial \mathfrak{L}_{t}}{\partial \eta}\right)\right)\right)+ \\
& \frac{1}{\sqrt{|h|}}\left(\frac{\partial}{\partial X^{\alpha}}\left(\sqrt{|h|}\left[\frac{\partial \mathfrak{L}}{\partial \xi_{\alpha}}\right]_{t}\right)-a d_{\xi_{\alpha}}^{*}\left(\sqrt{|h|}\left[\frac{\partial \mathfrak{L}}{\partial \xi_{\alpha}}\right]_{t}\right)\right)=F_{\text {ext }, t} \\
& \left(\frac{|h|}{|\bar{h}|}\right)^{1 / 2}\left[\frac{\partial \mathfrak{L}}{\partial \xi_{\alpha}}\right]_{t} \nu_{\alpha}=-\bar{F}_{\text {ext }, t},
\end{aligned}
$$

Let us do several remarks after these two sets of equations.

Remark 5.1: The new equations (43) and (44) prolong to the physical space, the equations (24) of [11, which were related to the material non-metric space $\mathcal{D}$. As expected, they have the physical insights that were missing in the material ones (24). This will become more apparent in the subsequent sections where they will be related to continuous media mechanics, and especially, shell theory.

Remark 5.2: Equations (43) and (44) can be directly derived by re-starting the variational calculus leading to (24), but with Lagrangian and external forces densities per unit of metric-volume of reference and deformed configuration respectively. This calculation is done in Appendix 1 in the deformed configuration.

Remark 5.3: While in the reference configuration the density of space-conjugate momentum $\Lambda_{o}^{\alpha}$ directly derives from the Lagrangian density $\mathfrak{L}_{o}$, this is not the case in the deformed configuration for which $\Lambda_{t}^{\alpha}$ is related to $\mathfrak{L}_{t}$ through:

$$
\Lambda_{t}^{\alpha}=-\left[\frac{\partial \mathfrak{L}}{\partial \xi_{\alpha}}\right]_{t}=-\left(\frac{\partial \mathfrak{L}_{t}}{\partial \xi_{\alpha}}\right)-\left(\begin{array}{c}
0 \\
\Gamma^{\alpha}
\end{array}\right) \mathfrak{L}_{t},
$$

where we used the fact that $\partial \sqrt{|h|} / \partial \Gamma_{\alpha}=\sqrt{|h|} \Gamma^{\alpha}$. Note that $(45)$ makes appear a convective term (induced by the current deformation of $\mathcal{D}$ while the Lagrangian density $\mathfrak{L}_{t}$ is kept constant). Referring to three-dimensional hyperelasticity, this corresponds to the well known fact that an Eulerian stress tensor, as the Cauchy stress tensor, has no conjugate strain field, and that consequently there is no pure Eulerian hyperelastic constitutive law. Note that this context can be easily circumvented, and the convective term of 45) removed, if instead of taking a 
density of strain energy $\mathfrak{U}_{t}$, we use a $\mathfrak{U}_{o}$ related to the reference configuration, and the mixed (Lagrangian-Eulerian) constitutive law:

$$
\Lambda_{t}^{\alpha}=-\left[\frac{\partial \mathfrak{L}}{\partial \xi_{\alpha}}\right]_{t}=\left|\frac{h_{o}}{h}\right|^{\frac{1}{2}}\left(\frac{\partial \mathfrak{U}_{o}}{\partial \xi_{\alpha}}\right),
$$

which has to be inserted into equations (44).

Remark 5.4: In (43), since $\left|h_{o}\right|$ is time-independent, we can remove it from the top line related to the time dimension. This elimination cannot be applied to the first line of (44). However, invoking the conservation of mass, allows this expression to be simplified in a way we will detail in section 7 , when we will apply these equations to classical Cosserat shells.

Remark 5.5: We recognize in (24), $(43)$ and $(44)$ the divergence of the fields $\Lambda^{\alpha} E_{\alpha}, \Lambda_{o}^{\alpha} h_{o \alpha}$ and $\Lambda_{t}^{\alpha} h_{\alpha}$ in the material chart of $\mathcal{D}$ and in its curvilinear deformation by $\Phi_{o} \circ e$ and $\Phi_{t} \circ e$ respectively, i.e.:

$$
\begin{gathered}
D I V\left(\left(\frac{\partial \mathfrak{L}}{\partial \xi_{\alpha}}\right) \otimes E_{\alpha}\right)=\frac{\partial}{\partial X^{\alpha}}\left(\frac{\partial \mathfrak{L}}{\partial \xi_{\alpha}}\right), \\
D I V\left(\left(\frac{\partial \mathfrak{L}_{o}}{\partial \xi_{\alpha}}\right) \otimes h_{o \alpha}\right)=\frac{1}{\sqrt{\left|h_{o}\right|}} \frac{\partial}{\partial X^{\alpha}}\left(\sqrt{\left|h_{o}\right|}\left(\frac{\partial \mathfrak{L}_{o}}{\partial \xi_{\alpha}}\right)\right), \\
D I V\left(\left[\frac{\partial \mathfrak{L}}{\partial \xi_{\alpha}}\right]_{t} \otimes h_{\alpha}\right)=\frac{1}{\sqrt{|h|}} \frac{\partial}{\partial X^{\alpha}}\left(\sqrt{|h|}\left[\frac{\partial \mathfrak{L}}{\partial \xi_{\alpha}}\right]_{t}\right) .
\end{gathered}
$$

Similarly, using the definitions of the unit normals to a material, reference, and deformed area element, as they are introduced in section 2.4, the boundary conditions of (24), (43) and (44) can be rewritten in the alternative forms, which handle fluxes of stress:

$$
\begin{gathered}
\left(\left(\frac{\partial \mathfrak{L}}{\partial \xi_{\alpha}}\right) \otimes E_{\alpha}\right) \cdot \nu=-\bar{F}_{e x t} \\
\left(\left(\frac{\partial \mathfrak{L}_{o}}{\partial \xi_{\alpha}}\right) \otimes h_{o \alpha}\right) \cdot \nu_{o}=-\bar{F}_{e x t, o} \\
\left(\left[\frac{\partial \mathfrak{L}}{\partial \xi_{\alpha}}\right]_{t} \otimes h_{\alpha}\right) \cdot \nu_{t}=-\bar{F}_{e x t, t} .
\end{gathered}
$$

Note that from top to bottom, the three divergence operators of 4749 and the corresponding boundary conditions 50 naturally appear through the application of the divergence theorem, when deriving (24), (43) and (44) from the Hamilton principle (see remark 5.2 and Appendix 1). Note also that these three operators can be extended to space-time by taking the metric $g_{i j}\left(g^{i} \otimes g^{j}\right)$, $i, j=0,1,2 . . p$, with $g_{00}=1, g_{0 \alpha}=0, g_{\alpha \beta}=h_{\alpha \beta}, \alpha, \beta=1,2 \ldots p$. Finally, these operators and the associated boundary conditions underly a model of stress for 
Cosserat media which is detailed in the next section.

Remark 5.6: Equations (44), as (43) and (24), represent the reduced dynamics of a Cosserat medium in the material frame. When $F_{\text {ext, } t}$ is g-independent (i.e., when the external forces are left invariant), these equations are first-order partial differential equations governing the material velocity field of the medium that can be integrated separately from the reconstruction of its configuration. This decoupling between dynamics and kinematics is the consequence of the symmetry properties (left invariance) of the Lagrangian density in the material setting. Alternatively, we knows from Noether theorem that symmetries are also associated with conservation laws. This second point of view on the above reduction appears if one chooses to express (44) (or 24), (43)) in the fixed frame $\left(o, e_{1}, e_{2}, e_{3}\right)$ of space. To perform this change from the material to the spatial setting, it suffices to exploit the properties of the co-adjoint action of $G$ on $\mathfrak{g}^{*}$, and to rewrite 44 in the equivalent form:

$$
\begin{aligned}
\frac{\partial}{\partial t}\left(A d_{\mathbf{g}^{-1}}^{*}\left(\sqrt{|h|}\left(\frac{\partial \mathfrak{L}_{t}}{\partial \eta}\right)\right)\right) & +\frac{\partial}{\partial X^{\alpha}}\left(A d_{\mathrm{g}^{-1}}^{*}\left(\sqrt{|h|}\left[\frac{\partial \mathfrak{L}}{\partial \xi_{\alpha}}\right]_{t}\right)\right) \\
& =A d_{\mathbf{g}^{-1}}^{*}\left(\sqrt{|h|} F_{\text {ext }, t}\right),
\end{aligned}
$$

where we introduced the further densities of spatial kinetic, stress, and external wrenches per unit of deformed volume, all the wrenches being related to the fixed frame $\left(o, e_{1}, e_{2}, e_{3}\right)$ :

$$
A d_{\mathrm{g}^{-1}}^{*}\left(\frac{\partial \mathfrak{L}_{t}}{\partial \eta}\right), A d_{\mathrm{g}^{-1}}^{*}\left[\frac{\partial \mathfrak{L}}{\partial \xi_{\alpha}}\right]_{t}, A d_{\mathrm{g}^{-1}}^{*} F_{e x t, t} .
$$

Physically, equation $(53)$ (which has its counterpart on $\mathcal{D}$ and $\left.\left(\Phi_{o} \circ e\right)(\mathcal{D})\right)$ stands for the spatial Poincaré equations of a Cosserat medium in the "conservation form". In particular, if the volume external forces are zero (i.e. $F_{\text {ext }, t}=0$ ), (53) can be interpreted as a zero-divergence condition in $\mathcal{D} \times \mathbb{R}^{+}$(with the extended metric mentioned by remark 5.5), as it is expected from Noether theorem in the context of field theory. In the language of gauge theory, this is the local conservation law (or continuity equation) of the generalized momentums in space-time. Using divergence theorem, this zero-divergence condition can be changed into a zero-flux condition, which when stated on the boundaries of $\mathcal{D} \times[0, t]$ with $\bar{F}_{e x t, t}=0$, leads to the global conservation law:

$$
\left(\int_{\mathcal{D}} A d_{\mathrm{g}^{-1}}^{*}\left(\frac{\partial \mathfrak{L}_{t}}{\partial \eta}\right) \sqrt{|h|} d \mathcal{D}\right)(t)=\left(\int_{\mathcal{D}} A d_{\mathbf{g}^{-1}}^{*}\left(\frac{\partial \mathfrak{L}_{t}}{\partial \eta}\right) \sqrt{|h|} d \mathcal{D}\right)(t=0),
$$

which mechanically stands for the conservation of the total kinetic wrench of the Cosserat medium along time. Note that being based on a zero-flux condition, this conservation law holds for any compact manifold $\left(\Phi_{o} \circ e\right)(\mathcal{D})$ without boundary. In particular, for mono-dimensional media (i.e. $p=1)$, (55) can be interpreted as the conservation of the circulation of the spatial angular momentum along any Cosserat beam closed in a loop [18. In this alternative formulation, (55) 
becomes a consequence of the more general Kelvin-Noether theorem which also holds for the ideal fluid 34 .

\subsection{Model of Cosserat stress}

To relate the model of stress underlaid by the Poincaré equations $(24),(43),(44)$ to the stress' model of mechanics of continuous media, we consider $G=S E(3)$ represented by matrices (5), and identify $\mathfrak{g}=s e(3)$ with $\mathbb{R}^{6}$ endowed with its six dimensional cross product [35], with basis $\left\{\left(E_{i}, 0\right),\left(0, E_{i}\right)\right\}_{i=1,2,3}$ where 0 and $E_{i}$ are the zero and basis vectors of $\mathcal{B} \simeq \mathbb{R}^{3}$. Moreover, using the metric of $\mathbb{R}^{6}$ allows us to identify $s e(3)^{*}$ to $s e(3)$ and to rewrite $(30 \mathrm{~b})$ and $(31 \mathrm{~b})$ in the equivalent form:

$$
\begin{aligned}
& \Upsilon_{t}=\left(\begin{array}{c}
\Omega_{i} E^{i} \\
V_{i} E^{i}
\end{array}\right) \otimes d t+\left(\begin{array}{c}
K_{i \alpha} E^{i} \\
\Gamma_{i \alpha} E^{i}
\end{array}\right) \otimes h^{\alpha}, \\
& \Lambda_{t}=\left(\begin{array}{c}
\Sigma_{t}^{i} E_{i} \\
P_{t}^{i} E_{i}
\end{array}\right) \otimes \frac{\partial}{\partial t}+\left(\begin{array}{c}
M_{t}^{i \alpha} E_{i} \\
N_{t}^{i \alpha} E_{i}
\end{array}\right) \otimes h_{\alpha} .
\end{aligned}
$$

The two tensor fields $\Upsilon_{t}$ and $\Lambda_{t}$ not only act on each other through the duality product $(<., .>)$, but also operate on $T\left(\Phi_{t} \circ e\right)(\mathcal{D}) \times \mathbb{R}^{+}$and its dual respectively. In particular, the time (0)-components of $(56)$ operate as follows on the vectors and co-vectors of the time axis:

$$
\begin{aligned}
& \left(\begin{array}{c}
\Omega \\
V
\end{array}\right) \otimes d t(\partial / \partial t)=\left(\begin{array}{c}
\Omega \\
V
\end{array}\right), \\
& \left(\begin{array}{c}
\Sigma_{t} \\
P_{t}
\end{array}\right) \otimes(\partial / \partial t)(d t)=\left(\begin{array}{c}
\Sigma_{t} \\
P_{t}
\end{array}\right) .
\end{aligned}
$$

In the same way, the space $(\alpha)$-components of (56) operate on the vectors $d X^{\beta} h_{\beta}$ and the co-vectors $d S_{t} \nu_{t, \beta} h^{\beta}=d S_{t} \nu_{t}$ of 12 respectively, as follows:

$$
\begin{aligned}
& \left(\begin{array}{c}
K_{\alpha} \\
\Gamma_{\alpha}
\end{array}\right) \otimes h^{\alpha} \cdot\left(d X^{\beta} h_{\beta}\right)=\left(\begin{array}{c}
K_{\beta} \\
\Gamma_{\beta}
\end{array}\right) d X^{\beta}, \\
& \left(\begin{array}{c}
M_{t}^{\alpha} \\
N_{t}^{\alpha}
\end{array}\right) \otimes h_{\alpha} \cdot\left(d S_{t} \nu_{t, \beta} h^{\beta}\right)=\left(\begin{array}{c}
M_{t}^{\beta} \\
N_{t}^{\beta}
\end{array}\right) \nu_{t, \beta} d S_{t} .
\end{aligned}
$$

The operations $57 \mathrm{a}$ and $\left(58 \mathrm{a}\right.$, give the rate of rotation $R^{T} . d R$ (of axis) and displacement $R^{T} \cdot d r$ (of the base point) of the orthonormal mobile spatial frames $\left(R(\bar{X}, t) \cdot E_{i}\right)=\left(t_{i}\right)_{i=1,2,3}(\bar{X}, t)$ due to a small time variation $(57)$, and a small displacement $d X^{\beta} h_{\beta}$ along $\left(\Phi_{t} \circ e\right)(\mathcal{D})(58)$ (see figure 1). In particular, note that the vectors $\Gamma_{\alpha}$ are those defined in section 2.4 as the pulled back of the convected basis $\left\{h_{\alpha}\right\}_{\alpha=1,2 . . p}$ in the material frame. The operation $(57 \mathrm{~b})$ gives the density of kinetic wrench per unit of deformed volume of $\left(\Phi_{t} \circ e\right)(\mathcal{D})$ at time $t$. The operation $(58 \mathrm{~b})$ models the material resultant $\left(d N_{i n t}\right)$, and the material moment $\left(d M_{i n t}\right)$ of internal contact forces exerted across an oriented deformed 
surface element $d S_{t} \nu_{t}=d S_{t} \nu_{t, \beta} h^{\beta}$ of $\left(\Phi_{t} \circ e\right)(\mathcal{D})$ by the piece of deformed material toward which $\nu_{t}$ points, onto its complement part (see figure 3 in the case of a shell). Repeating this context on $\mathcal{D}$ and $\left(\Phi_{o} \circ e\right)(\mathcal{D})$, i.e. by using $(2728)$ and (30a, 31a respectively instead of $30 \mathrm{~b}, 31 \mathrm{~b})$, allows us to introduce three tensor density fields of Cosserat stress whose components are related to the space $(\alpha)$ conjugate momenta of (24), (43) and (44) as follows:

$$
\begin{aligned}
& \left(\begin{array}{c}
M^{\alpha} \\
N^{\alpha}
\end{array}\right) \otimes E_{\alpha}=-\left(\frac{\partial \mathfrak{L}}{\partial \xi_{\alpha}}\right) \otimes E_{\alpha}, \\
& \left(\begin{array}{c}
M_{o}^{\alpha} \\
N_{o}^{\alpha}
\end{array}\right) \otimes h_{o \alpha}=-\left(\frac{\partial \mathfrak{L}_{o}}{\partial \xi_{\alpha}}\right) \otimes h_{o \alpha}, \\
& \left(\begin{array}{c}
M_{t}^{\alpha} \\
N_{t}^{\alpha}
\end{array}\right) \otimes h_{\alpha}=-\left[\frac{\partial \mathfrak{L}}{\partial \xi_{\alpha}}\right]_{t} \otimes h_{\alpha} .
\end{aligned}
$$

It is worth noting here that these three densities of tensor are consistent with the three divergence operators and boundary conditions 47450, (48) 51), 49, 42 of remark 5.5. The first (59a) (respectively, second (59b) and third (59c)) of these three tensor-densities, allows defining the density of internal stress wrench $F_{\text {int }}=$ $\left(M_{i n t}^{T}, N_{i n t}^{T}\right)^{T}$ exerted across an oriented material surface element (respectively, a reference and deformed surface element) of normal $\nu$ (respectively, of normal $\nu_{o}$ and $\nu_{t}$ ), by the piece of material (respectively, the piece of reference and deformed configuration) toward which $\nu$, (respectively $\nu_{o}$ and $\nu_{t}$ ) points, per unit of its material (respectively per unit of reference and deformed) area, as follows:

$$
\frac{d F_{\text {int }}}{d S}=\left(\begin{array}{c}
M^{\beta} \\
N^{\beta}
\end{array}\right) \nu_{\beta}, \frac{d F_{\text {int }}}{d S_{o}}=\left(\begin{array}{c}
M_{o}^{\beta} \\
N_{o}^{\beta}
\end{array}\right) \nu_{o, \beta}, \frac{d F_{\text {int }}}{d S_{t}}=\left(\begin{array}{c}
M_{t}^{\beta} \\
N_{t}^{\beta}
\end{array}\right) \nu_{t, \beta} .
$$

Remark 5.7: The above stress tensors have their left leg in the material frame. Referring to section 2.4, we can push forward them from the material to the microstructure frames to define the three spatial tensor fields:

$$
\begin{aligned}
& \left(\begin{array}{c}
m \\
n
\end{array}\right)=\left(\begin{array}{c}
m^{\alpha} \\
n^{\alpha}
\end{array}\right) \otimes E_{\alpha}, \\
& \left(\begin{array}{c}
m_{o} \\
n_{o}
\end{array}\right)=\left(\begin{array}{c}
m_{o}^{\alpha} \\
n_{o}^{\alpha}
\end{array}\right) \otimes h_{o \alpha}, \\
& \left(\begin{array}{c}
m_{t} \\
n_{t}
\end{array}\right)=\left(\begin{array}{c}
m_{t}^{\alpha} \\
n_{t}^{\alpha}
\end{array}\right) \otimes h_{\alpha},
\end{aligned}
$$

where $m_{., o, t}^{\alpha}=R \cdot M_{\cdot, o, t}^{\alpha}=M_{., o, t}^{i \alpha} t_{i}$ and $n_{., o, t}^{\alpha}=R \cdot N_{., o, t}^{\alpha}=N_{., o, t}^{i \alpha} t_{i}$ are densities of spatial forces and couples per unit of material, reference and deformed volume. These three tensor-densities map a material 61a), a reference 61b), and a deformed $61 \mathrm{c}$ ) oriented area element subscripted with ., $o, t$ respectively, onto the spatial internal wrench $d f_{i n t}=\left(d m_{i n t}^{T}, d n_{i n t}^{T}\right)^{T} \in \mathfrak{g}^{*}$ exerted on it. Referring to elasticity of classical 3D media, the tensor $61 \mathrm{~b}$ generalizes the first Piola-Kirchhoff since it acts on the reference area element to give the resultant 
of internal contact forces and couples on the deformed configuration. Similarly, the tensor (61c) defines a pure Eulerian tensor stress (as the Cauchy tensor is) acting on the deformed area element to give the resultant of internal contact forces and couples exerted on it in the deformed configuration.

Remark 5.8: In the same line, let us reconsider the space $(\alpha)$-components of (58a) as well as their counterparts in the material space and the reference configuration, and let us push-forward their left leg from the material to the microstructure frames, one can introduce the following strain tensors:

$$
\left(\begin{array}{c}
k \\
\gamma
\end{array}\right)=\left(\begin{array}{c}
k_{\alpha} \\
\gamma_{\alpha}
\end{array}\right) \otimes E^{\alpha},\left(\begin{array}{c}
k_{o} \\
\gamma_{o}
\end{array}\right)=\left(\begin{array}{c}
k_{\alpha} \\
\gamma_{\alpha}
\end{array}\right) \otimes h_{o}^{\alpha},\left(\begin{array}{c}
k_{t} \\
\gamma_{t}
\end{array}\right)=\left(\begin{array}{c}
k_{\alpha} \\
\gamma_{\alpha}
\end{array}\right) \otimes h^{\alpha},
$$

where $\gamma_{\alpha}=\Gamma_{i \alpha} t^{i}=\partial r / \partial X^{\alpha}$ and $k_{\alpha}=K_{i \alpha} t^{i}=\left(\left(\partial R / \partial X^{\alpha}\right) \cdot R^{T}\right)^{\vee}$, with $\vee$ changing a skew symmetric tensor of $\mathbb{R}^{3} \otimes \mathbb{R}^{3}$ into its axial vector in $\mathbb{R}^{3}$. We recognize in $\Gamma_{i \alpha} t^{i} \otimes h_{o}^{\alpha}$ the expression of the gradient of the transformation restricted to $\left(\Phi_{o} \circ e\right)(\mathcal{D})$, i.e., $\bar{F} \triangleq h_{\alpha} \otimes h_{o}^{\alpha}$, while $K_{i \alpha} t^{i} \otimes h_{o}^{\alpha}$ represents the gradient of the rotation of the microstructures, named wryness tensor in [1]. The three pairs of tensors: $\left(k_{t}, \gamma_{t}\right)$ and $\left(m_{t}, n_{t}\right),\left(k_{o}, \gamma_{o}\right)$ and $\left(m_{o}, n_{o}\right)$, and $(K, \Gamma)$ and $\left(M_{t}, N_{t}\right)$, are dual of each other, as are the first Piola-Kirchhoff stress tensor and the gradient of transformation in the case of $3 \mathrm{D}$ media [33. Furthermore for a classical (not micropolar) medium $\mathcal{B}$, using the general Cosserat Kinematics (8), we have the following expression of the virtual work of the internal forces exerted inside $\mathcal{B}$ :

$$
\delta W_{\text {int }}=\int_{\mathcal{B}} P: \delta F \sqrt{\left|g_{o}\right|} d \mathcal{B}=\int_{\mathcal{D}}\left(M_{t}^{\alpha} \cdot \delta K_{\alpha}+N_{t}^{\alpha} \cdot \delta \Gamma_{\alpha}\right) \sqrt{|h|} d \mathcal{D},
$$

where $F$ and $P$ are the 3D-gradient of transformation and the 3D-first Piola Kirchhoff stress tensor between $\Phi_{o}(\mathcal{B})$ and $\Phi_{t}(\mathcal{B})$, while $\sqrt{\left|g_{o}\right|}$ is the density of metric volume on $\Phi_{o}(\mathcal{B})$. This general expression is derived in Appendix 2 .

\section{Application to full Cosserat shells: Geometrically exact model of micropolar shells}

In this section we consider the case of shells having an intrinsic spin and couple stress around their directors, or "micropolar" shells. In this nominal context, the full Cosserat-Poincaré construction applies. In section 7, we will show how this model can be adapted to classical shells, i.e. shells with no intrinsic spin nor couple stress around their directors.

\subsection{Full Cosserat shells kinematics}

In the case of shells, the Cosserat micro-structure $\mathcal{M}$ models rigid fibers (supported by $E_{3}$ ), traversing the shell's material mid surface $\mathcal{D}$ (supported by $\left.\left(E_{1}, E_{2}\right)\right)$. They are labelled by $\left(X^{1}, X^{2}\right)$, two parameters which define a set 


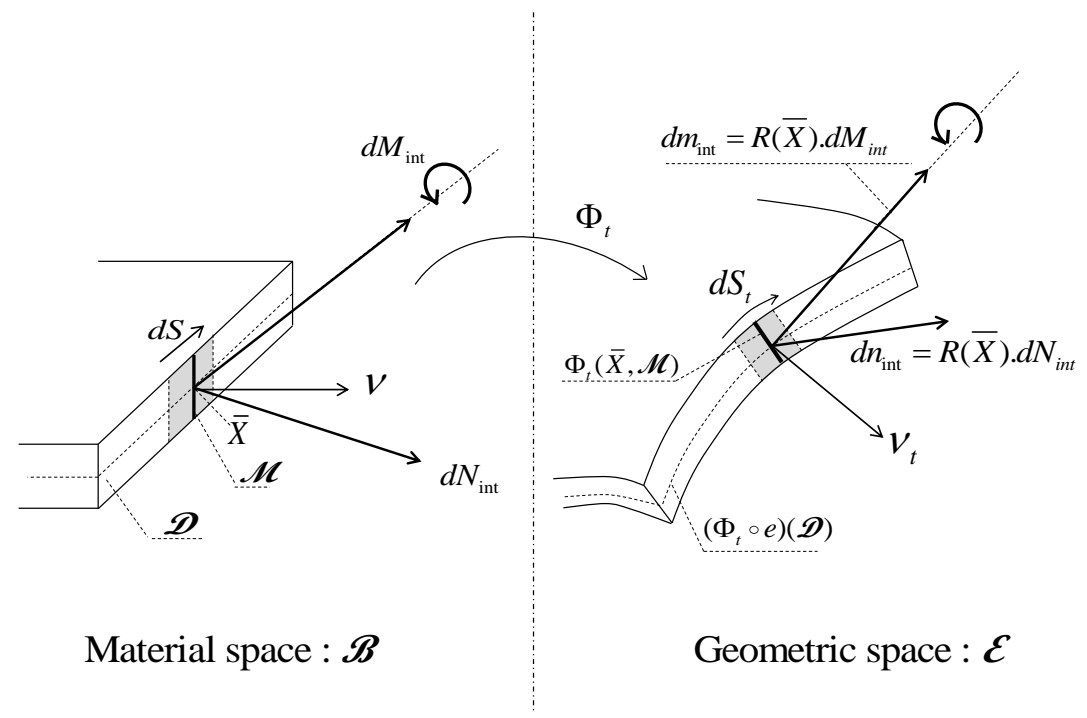

Fig. 3. Picture of the stress in Cosserat shells: $d m_{\text {int }}$ and $d n_{\text {int }}$ define the spatial wrench of internal contact forces exerted through the element $d S_{t}$ from right to left.

of coordinates on $\mathcal{D}$. The configuration space of one such rigid microstructure, say that labelled by $\left(X^{1}, X^{2}\right)$, is the set of transformations $\mathrm{g}\left(X^{1}, X^{2}\right) \in S E(3)$, and the whole configuration space of the shell is the space of the $\left(X^{1}, X^{2}\right)$ parameterized surfaces in $S E(3)$ :

$$
C:=\left\{\mathrm{g}:\left(X^{1}, X^{2}\right) \in[0,1]^{2} \mapsto \mathrm{g}\left(X^{1}, X^{2}\right) \in S E(3)\right\} .
$$

Here, with no restriction, $\left(X^{1}, X^{2}\right)$ are normalized on $\mathcal{D}$. Note that this definition appears in the following Reissner shells kinematics [23, which instantiates the general kinematics (8) for $p=2$ :

$$
\Phi(X)=r\left(X^{1}, X^{2}\right)+R\left(X^{1}, X^{2}\right) \cdot\left(X^{3} E_{3}\right) .
$$

Let us recall that $\left(O, E_{1}, E_{2}, E_{3}\right)$ interchangeably defines the material or inertial frame, to which $r$ and $R$ are related. With this definition of the shell configuration space, the left invariant fields of the general construction are:

$$
\eta=\mathrm{g}^{-1} \frac{\partial \mathrm{g}}{\partial t}=\left(\begin{array}{cc}
\Omega & V \\
0 & 0
\end{array}\right), \xi_{\alpha}=\mathrm{g}^{-1} \frac{\partial \mathrm{g}}{\partial X^{\alpha}}=\left(\begin{array}{cc}
K_{\alpha} & \Gamma_{\alpha} \\
0 & 0
\end{array}\right), \alpha=1,2 .
$$

where $\Omega\left(X^{1}, X^{2}\right)$ and $V\left(X^{1}, X^{2}\right)$ denote the linear and angular velocities of the $\left(X^{1}, X^{2}\right)$-rigid fiber pulled back in the material frame. Similarly, $\left(K_{1}, K_{2}\right)$ and 
$\left(\Gamma_{1}, \Gamma_{2}\right)$ are in the same frame and stand for the $X^{1}$ and $X^{2}$-rates of rotation and position of the mobile frames $\left(t_{i}\right)_{i=1,2,3}\left(X^{1}, X^{2}\right)$. According to $\left.30 \mathrm{~b}\right)$, all the vector fields of 65 define the components of the unique 1-form:

$$
\Upsilon_{t}=\left(\begin{array}{c}
\Omega \\
V
\end{array}\right) \otimes d t+\left(\begin{array}{c}
K_{\alpha} \\
\Gamma_{\alpha}
\end{array}\right) \otimes h^{\alpha}
$$

which maps vectors of $T\left(\left(\Phi_{t} \circ e\right)(\mathcal{D}) \times \mathbb{R}^{+}\right)$to space-time rate vectors of $\mathfrak{g}$.

\subsection{Conservation of mass for shells}

The conservation of mass will be used in the following to recover the geometrically exact balances of classical shells in the deformed configuration from the Poincaré equations (44). To state it, it is sufficient to note that the mass and inertia tensor of the microstructures above $\mathcal{D}$ define some volume densities which transform according to [12]:

$$
\bar{\rho}=\bar{\rho}_{o} \sqrt{\left|h_{o}\right|}=\bar{\rho}_{t} \sqrt{|h|}, \bar{J}=\bar{J}_{o} \sqrt{\left|h_{o}\right|}=\bar{J}_{t} \sqrt{|h|}
$$

where we introduced the mass-densities $\bar{\rho}, \bar{\rho}_{o}$ and $\bar{\rho}_{t}$ (respectively, the angular inertia tensor-densities $\bar{J}, \bar{J}_{o}$ and $\bar{J}_{t}$ ) per unit of material, reference and deformed volume. The two first being function in $\bar{X}$, while the third depends on both, space and time.

\subsection{Model of the conjugate momentums for full-Cosserat shells}

Based on the previous kinematics and the frame indifference of finite elasticity [29], we consider a reduced shell's Lagrangian volume form of the form:

$$
\left(\mathfrak{T}_{t}(\eta)-\mathfrak{U}_{t}\left(\xi_{1}, \xi_{2}\right)\right) \sqrt{|h|} d X^{1} \wedge d X^{2} \in \wedge^{2} T_{X}^{*}(\mathcal{D}),
$$

where $\mathfrak{T}_{t}$ and $\mathfrak{U}_{t}$ denote the (left-reduced) kinetic and elastic potential energy densities, with $\mathfrak{L}_{t}=\mathfrak{T}_{t}-\mathfrak{U}_{t}$ the reduced density of Lagrangian, all these densities being measured per unit of deformed volume. Introducing (68) in the general Cosserat equations on the deformed configuration (44, with $p=2$ makes the

fields of densities of wrenches per unit of metric area of deformed mid-surface shell appear:

$$
\begin{aligned}
& \frac{\partial \mathfrak{T}_{t}}{\partial \eta}=\left(\begin{array}{c}
\partial \mathfrak{T}_{t} / \partial \Omega \\
\partial \mathfrak{T}_{t} / \partial V
\end{array}\right)=\left(\begin{array}{c}
\Sigma_{t} \\
P_{t}
\end{array}\right), \\
&\left(\begin{array}{c}
\partial \mathfrak{U}_{t} / \partial K_{\alpha} \\
\partial \mathfrak{U}_{t} / \partial \Gamma_{\alpha}
\end{array}\right)-\left(\begin{array}{c}
0 \\
\Gamma^{\alpha}
\end{array}\right) \mathfrak{L}_{t}=\left(\begin{array}{c}
M_{t}^{\alpha} \\
N_{t}^{\alpha}
\end{array}\right) .
\end{aligned}
$$

Note that (69) defines a generalized (space-time) constitutive law of the full Cosserat model of hyperelastic shells. Furthermore, according to $56 \mathrm{~b}, 6$, 69 geometrically defines the unique tensor density field per unit of deformed volume:

$$
\Lambda_{t}=\left(\begin{array}{c}
\Sigma_{t} \\
P_{t}
\end{array}\right) \otimes \frac{\partial}{\partial t}+\left(\begin{array}{c}
M_{t}^{\alpha} \\
N_{t}^{\alpha}
\end{array}\right) \otimes h_{\alpha}
$$


Assuming that the material mid-surface $\mathcal{D}$ crosses the microstructure $\mathcal{M}$ in its geometric center which coincides with its mass center, and positioning the material frame in this point, the shell's kinetic energy density per unit of deformed mid-surface area is:

$$
\mathfrak{T}_{t}(\eta)=\frac{1}{2}<\left(\begin{array}{c}
\Omega \\
V
\end{array}\right),\left(\begin{array}{c}
\bar{J}_{t} \Omega \\
\bar{\rho}_{t} V
\end{array}\right)>,
$$

with $\bar{\rho}_{t}$ and $\bar{J}_{t}$, the mass and the angular inertia tensor density per unit of volume of $\left(\Phi_{t} \circ e\right)(D)$ defined as $\bar{\rho}_{t}=\bar{\rho} / \sqrt{h}$ and $\bar{J}_{t}=\bar{J} / \sqrt{h}$, with $\bar{\rho}$ and $\bar{J}=$ $\bar{j}_{\perp}\left(E_{1} \otimes E^{1}+E_{2} \otimes E^{2}\right)+\bar{j}_{\|} E_{3} \otimes E^{3}=\bar{J}_{\perp}+\bar{J}_{\|}$, the mass and the inertia tensor of the microstructure $\mathcal{M}$. With (71), the density of kinetic wrench per unit of current mid-surface area is:

$$
\frac{\partial \mathfrak{T}_{t}}{\partial \eta}=\left(\begin{array}{c}
\Sigma_{t} \\
P_{t}
\end{array}\right)=\left(\begin{array}{c}
\bar{J}_{t} \Omega \\
\bar{\rho}_{t} V
\end{array}\right) .
$$

It is worth noting here that $\mathcal{M}$ is assumed to have an intrinsic spin $\left(\bar{j}_{\|} / \sqrt{|h|}\right) \Omega_{3}$ about its axis, an assumption which will be relaxed in the classical shell model in section 7 .

\subsection{Model of stress for full Cosserat shells}

According to the Cosserat stress picture in section 4, the $X^{\alpha}$-terms of $\Lambda_{t}$, with $\Lambda_{t}$ detailed as in $(70)$, define two densities of tensors per unit of deformed midsurface area: $N_{t}=N_{t}^{\alpha} \otimes h_{\alpha}=N_{t}^{i \alpha} E_{i} \otimes h_{\alpha}$ and $M_{t}=M_{t}^{\alpha} \otimes h_{\alpha}=M_{t}^{i \alpha} E_{i} \otimes h_{\alpha}$, which operate as follows on co-vectors of $T^{*} \Phi_{t}(e(\mathcal{D}))$, here standing for the deformed surface elements $\nu_{t} d S_{t}$, with $\nu_{t}$ the unit normal to any convected line cutting $\mathcal{D}$ :

$$
\begin{gathered}
\left(N_{t}^{i \beta} E_{i} \otimes h_{\beta}\right) \cdot\left(\nu_{t \alpha} h^{\alpha} d S_{t}\right)=N_{t}^{i \alpha} E_{i} \nu_{t \alpha} d S_{t}=N_{t}^{\alpha} \nu_{t \alpha} d S_{t}, \\
\left(M_{t}^{i \beta} E_{i} \otimes h_{\beta}\right) .\left(\nu_{t \alpha} h^{\alpha} d S_{t}\right)=M_{t}^{i \alpha} E_{i} \nu_{t \alpha} d S_{t}=M_{t}^{\alpha} \nu_{t \alpha} d S_{t} .
\end{gathered}
$$

From (60), $N_{t}^{\alpha} \nu_{t \alpha} d S_{t}$ and $M_{t}^{\alpha} \nu_{t \alpha} d S_{t}$ represent the resultant of internal contact forces and couples exerted on the deformed cross-sectional line of normal $\nu_{t \alpha} h^{\alpha}$ per unit of its metric length. More particularly, in agrement with shell literature [24, [25], remarking from section 2.4, that $\nu_{t, \alpha}=(|h| /|\bar{h}|)^{1 / 2} \nu_{\alpha}$, we can infer that $\left(|h| /\left|\overline{h_{2}}\right|\right)^{1 / 2} N_{t}^{1}$ and $\left(|h| /\left|\overline{h_{2}}\right|\right)^{1 / 2} M_{t}^{1}$ (respectively $\left(|h| /\left|\overline{h_{1}}\right|\right)^{1 / 2} N_{t}^{2}$ and $\left.\left(|h| /\left|\overline{h_{1}}\right|\right)^{1 / 2} M_{t}^{2}\right)$ represent the resultant and the momentum of internal stress forces exerted across the deformed shell's transverse section $X^{1}=C$ (respectively $X^{2}=C$ ), per unit of metric length $\left|\overline{h_{2}}\right|$ along $h_{2}$ (respectively, per unit of $\left|\overline{h_{1}}\right|$ along $h_{1}$ ), by the piece of material $X^{1}>C$ (respectively $X^{2}>C$ ), onto the contiguous piece $X^{1}<C$ (resp. $X^{2}<C$ ). One can partition them according to:

$$
\begin{aligned}
& N_{t}=N_{t}^{i \alpha} E_{i} \otimes h_{\beta}=N_{t}^{\alpha \beta} E_{\alpha} \otimes h_{\beta}+N_{t}^{3 \beta} E_{3} \otimes h_{\beta}, \\
& M_{t}=M_{t}^{i \alpha} E_{i} \otimes h_{\beta}=M_{t}^{\alpha \beta} E_{\alpha} \otimes h_{\beta}+M_{t}^{3 \beta} E_{3} \otimes h_{\beta} .
\end{aligned}
$$


Physically, $N_{t}^{1 \alpha}$ and $N_{t}^{2 \alpha}$ (resp. $M_{t}^{1 \alpha}$ and $M_{t}^{2 \alpha}$ ) model the resultant stress (respectively couple stress) exerted perpendicularly to the microstructure $\mathcal{M}$, while $N_{t}^{3 \alpha}$ is the transverse shearing resultant stress aligned with $\mathcal{M}$. Finally, it is worth noting here that in the classical shell model that will be introduced later, the microstructure frames are replaced by single vectors or "directors", and the two components $M_{t}^{3 \alpha}$, which represent couple stress aligned with $\mathcal{M}$, are zero. Moreover, in the same model, the stress couples $M_{t}^{\alpha}$ are often replaced by the director stress couples $\widetilde{M}_{t}^{\alpha}$ defined by $M_{t}^{\alpha}=E_{3} \times \widetilde{M}_{t}^{\alpha}[25]$.

\subsection{Derivation of the geometrically exact dynamic balance of full-Cosserat shells from Poincaré equations}

Introducing (65), (70) and (72) into the Poincaré equations in the deformed configuration (44) with the expression of $a d^{*}$ on $s e(3) \cong \mathbb{R}^{6}$, gives the dynamic equations of a Cosserat shell in the deformed configuration and the material frame:

$$
\begin{aligned}
& \frac{1}{\sqrt{|h|}}\left(\frac{\partial \sqrt{|h|} P_{t}}{\partial t}\right)+\Omega \times P_{t}=\frac{1}{\sqrt{|h|}}\left(\frac{\partial \sqrt{|h|} N_{t}^{\alpha}}{\partial X^{\alpha}}\right)+K_{\alpha} \times N_{t}^{\alpha}+N_{e x t, t},(75) \\
& \frac{1}{\sqrt{|h|}}\left(\frac{\partial \sqrt{|h|} \Sigma_{t}}{\partial t}\right)+\Omega \times \Sigma_{t}=\frac{1}{\sqrt{|h|}}\left(\frac{\partial \sqrt{|h|} M_{t}^{\alpha}}{\partial X^{\alpha}}\right)+K_{\alpha} \times M_{t}^{\alpha}+\Gamma_{\alpha} \times N_{t}^{\alpha}+M_{e x t, t} .
\end{aligned}
$$

Pushing forward these equations from the material to the microstructures' frames, gives:

$$
\begin{aligned}
& \frac{1}{\sqrt{|h|}}\left(\frac{\partial \sqrt{|h|} p_{t}}{\partial t}\right)=\frac{1}{\sqrt{|h|}}\left(\frac{\partial \sqrt{|h|} n_{t}^{\alpha}}{\partial X^{\alpha}}\right)+n_{e x t, t} \\
& \frac{1}{\sqrt{|h|}}\left(\frac{\partial \sqrt{|h|} \sigma_{t}}{\partial t}\right)=\frac{1}{\sqrt{|h|}}\left(\frac{\partial \sqrt{|h|} m_{t}^{\alpha}}{\partial X^{\alpha}}\right)+\frac{\partial r}{\partial X^{\alpha}} \times n_{t}^{\alpha}+m_{e x t, t}
\end{aligned}
$$

where small characters denote spatial tensors, i.e., $p_{t}=R . P_{t}, \sigma_{t}=R . \Sigma_{t}, n_{t}^{\alpha}=$ $R . M_{t}^{\alpha}, m_{t}^{\alpha}=R \cdot M_{t}^{\alpha}, n_{e x t, t}=R . N_{e x t, t}$, and $m_{e x t, t}=R . M_{e x t, t}$, while $\partial r / \partial X^{\alpha}=$ $R \cdot \Gamma_{\alpha}$. Alternatively, if we apply the Poincaré-equations related to the reference configuration 43 that we push forward, we obtain the other set of equations:

$$
\begin{aligned}
& \frac{\partial p_{o}}{\partial t}=\frac{1}{\sqrt{\left|h_{o}\right|}}\left(\frac{\partial \sqrt{\left|h_{o}\right|} n_{o}^{\alpha}}{\partial X^{\alpha}}\right)+n_{e x t, o} \\
& \frac{\partial \sigma_{o}}{\partial t}=\frac{1}{\sqrt{\left|h_{o}\right|}}\left(\frac{\partial \sqrt{\left|h_{o}\right|} m_{o}^{\alpha}}{\partial X^{\alpha}}\right)+\frac{\partial r}{\partial X^{\alpha}} \times n_{o}^{\alpha}+m_{e x t, o},
\end{aligned}
$$

which handle densities related to the metric volume of the reference configuration, especially those of spatial kinetic resultant and momentum $\sigma_{o}=R . \Sigma_{o}$, $p_{o}=R . P_{o}$, with $\Sigma_{o}$ and $P_{o}$, the counterparts of $(72)$, on the reference configuration. Equations 75 and $(76)$ stand for the geometrically exact balance 
equations of full-Cosserat shells in the deformed configuration, in the material and spatial setting respectively, while (77) are their counterpart in the reference configuration, and in the spatial setting. They are known from shell literature and can be found in some treatise books as [26] where they are derived from Euler's theorems. All of them represent the left-reduced shell dynamics in the dual of the Lie algebra of $S E(3)$, and have to be supplemented with a reconstruction equation. For instance, 75 needs the material reconstruction equation:

$$
\forall\left(X^{1}, X^{2}\right) \in[0,1]^{2}: \frac{\partial}{\partial t}\left(\begin{array}{cc}
R & r \\
0 & 1
\end{array}\right)=\left(\begin{array}{cc}
R & r \\
0 & 1
\end{array}\right)\left(\begin{array}{cc}
\Omega & V \\
0 & 0
\end{array}\right)
$$

which stands for a kinematic model supplementing 75 into a set of timeevolution equations in the state space of the shell initialized with $\bar{X} \mapsto\left(\mathrm{g}_{o}, \eta_{o}\right)(\bar{X})$ at $t=0$.

Remark 6.1: Referring to Remark 5.6, the Euler-Poincaré equations 75) and (76) can be alternatively (and equivalently) rewritten as the following local conservation law of spatial wrench:

$\frac{\partial}{\partial t}\left(A d_{\mathbf{g}^{-1}}^{*}\left(\begin{array}{c}\sqrt{|h|} \Sigma_{t} \\ \sqrt{|h|} P_{t}\end{array}\right)\right)=\frac{\partial}{\partial X^{\alpha}}\left(A d_{\mathbf{g}^{-1}}^{*}\left(\begin{array}{c}\sqrt{|h|} M_{t}^{\alpha} \\ \sqrt{|h|} N_{t}^{\alpha}\end{array}\right)\right)+A d_{\mathbf{g}^{-1}}^{*}\left(\begin{array}{c}\sqrt{|h|} M_{e x t, t} \\ \sqrt{|h|} N_{e x t, t}\end{array}\right)$,

where in contrast to $(76)$, in the above equation, all momentums are related to a unique fixed point standing for the origin of $\left(o, e_{1}, e_{2}, e_{3}\right)$.

To conclude this section, a closed formulation for full-Cosserat shells consists of the definition of strains given by the space-component of (66), the constitutive law $(69 \mathrm{~b})$, along with the material balance equations $(75)$ or the spatial ones (76) (or their counterparts related to the reference configuration), their boundary conditions, and the reconstruction equation (78) or its spatial counterpart, depending whether one choose to write the balance equations in the material or the spatial setting respectively. Alternatively, in the spatial setting, the balance equations 76 can be replaced by the conservation law 79 .

\section{Reduction of the full-Cosserat shell model: Geometrically exact model of classical shells}

Despite its elegance, the above construction cannot be directly applied to the classical shell model. It would hold for a 2-D micropolar medium whose the micro-structure $\mathcal{M}$ has a full rank angular dynamics i.e., has a non negligible intrinsic spin, external couple and couple stress along $\mathcal{M}$, here modelled respectively by the components $\Sigma_{t}^{3}=\left(\bar{j}_{\|} / \sqrt{h}\right) \Omega_{3}, M_{e x t, t}^{3}, M_{t}^{31}$ and $M_{t}^{32}$ of 75 . However, the classical shell model has no such features and the full Cosserat model must be modified to remove these artifacts for classical shells. 


\subsection{Reduction of the kinematic and kinetic models}

To achieve this elimination in the above balance equations, we force $\bar{j}_{\|}=0$, and restrict the angular field to satisfy $\omega=t_{3} \times \partial t_{3} / \partial t$. Then, as announced in section 6.2 , using the conservation of mass (67) in equations $(76)$, allows changing them into:

$$
\begin{aligned}
& \bar{\rho}_{t} \frac{\partial v}{\partial t}=\frac{1}{\sqrt{|h|}}\left(\frac{\partial \sqrt{|h|} n_{t}^{\alpha}}{\partial X^{\alpha}}\right)+n_{e x t, t} \\
& \bar{I}_{t} \cdot\left(t_{3} \times \frac{\partial^{2} t_{3}}{\partial t^{2}}\right)=\frac{1}{\sqrt{|h|}}\left(\frac{\partial \sqrt{|h|} m_{t}^{\alpha}}{\partial X^{\alpha}}\right)+\frac{\partial r}{\partial X^{\alpha}} \times n_{t}^{\alpha}+m_{e x t, t}
\end{aligned}
$$

Or alternatively, 777 into:

$$
\begin{aligned}
& \bar{\rho}_{o} \frac{\partial v}{\partial t}=\frac{1}{\sqrt{\left|h_{o}\right|}}\left(\frac{\partial \sqrt{\left|h_{o}\right|} n_{o}^{\alpha}}{\partial X^{\alpha}}\right)+n_{e x t, o} \\
& \bar{I}_{o} \cdot\left(t_{3} \times \frac{\partial^{2} t_{3}}{\partial t^{2}}\right)=\frac{1}{\sqrt{\left|h_{o}\right|}}\left(\frac{\partial \sqrt{\left|h_{o}\right|} m_{o}^{\alpha}}{\partial X^{\alpha}}\right)+\frac{\partial r}{\partial X^{\alpha}} \times n_{o}^{\alpha}+m_{e x t, o},
\end{aligned}
$$

where we used the fact that $p_{o, t}=\bar{\rho}_{o, t} v$ and $\sigma_{o, t}=\bar{I}_{o, t} . \omega$ with $v=R . V$, $\omega=R . \Omega$, and $\bar{I}_{o, t}=R \cdot \bar{J}_{o, t} \cdot R^{T}$. Reconsidering $(78)$ with our restricted angular velocity field, equations $(80)$ and $(81)$ have to be supplemented with the spatial reconstruction equation:

$$
\forall\left(X^{1}, X^{2}\right) \in[0,1]^{2}: \frac{\partial}{\partial t}\left(\begin{array}{cc}
R & r \\
0 & 1
\end{array}\right)=\left(\begin{array}{cr}
\left(t_{3} \times \partial t_{3} / \partial t\right)^{\wedge} & v \\
0 & 0
\end{array}\right) \cdot\left(\begin{array}{cc}
R & r \\
0 & 1
\end{array}\right) .
$$

Equations (80) and (81) are well known in the shell literature as the geometrically exact shell equations in the current and reference configuration. They were so far derived from Newton's law and Euler's theorem either by directly using the Cosserat model [36, [37, [38] or indirectly from 3D elasticity [12, [39]. When investigated by the direct approach, the dynamics of the directors $t_{3}$ are the most of the time, directly derived on $S^{2}$ through an alternative set of angular equations deduced from (80)-bottom (or 81)-bottom) by cross multiplying them on the right by $t_{3}$.

\subsection{Reduction of the model of stress}

The equations $80,, 81$ ) and 82 take charge the degeneration of $\mathcal{M}$ into directors in the kinematic and kinetic models. It remains to achieve the same reduction but for the model of stress. To achieve this, we apply in this section the usual procedure leading to the symmetry of the Cauchy stress tensor of a classical (non-Cosserat) 3D medium to our full-Cosserat shell. We will first define a set of reduced strain measures adapted to the classical model, and by duality will introduce a reduced model of the stress, related to these strains through 
hyperelastic constitutive laws that force the symmetry of two tensor stress fields modelling the stress state in the shell's mid-surface. As in the three-dimensional case [33], these symmetry conditions will be proved to be equivalent to the angular balance equations along the microstructures which in the classical model are directors, i.e. degenerated rigid bodies with no intrinsic spin nor couple stress along them. At the end, this constitutive law will entirely take charge the reduction of the stress state and the equations (80), (81) and 82 will hold for classical shells, as soon as they will be used with this constitutive law.

Strains of the Classical shell model According to the general PoincaréCosserat picture, any set of strain measures properly invariant under rigid transformations, should depend on the left invariant fields $\xi_{1}$ and $\xi_{2}$ of (66) only. This basic fact can be easily verified in the case of classical shells with no couple stress along the directors. Using the field of frame and co-frame $\bar{X} \mapsto\left(h_{1}, h_{2}\right)(\bar{X})$ and $\bar{X} \mapsto\left(h^{1}, h^{2}\right)(\bar{X})$ on $\left(\Phi_{t} \circ e\right)(\mathcal{D})$ as they are defined in section 2.3 , one can parameterize the strain state of a classical shell with the following set of tensor fields [12:

$$
\begin{gathered}
\epsilon=\epsilon_{\alpha \beta} h^{\alpha} \otimes h^{\beta}=\frac{1}{2}\left(h_{\alpha} \cdot h_{\beta}-h_{\alpha}^{o} \cdot h_{\beta}^{o}\right) h^{\alpha} \otimes h^{\beta}, \\
\rho=\rho_{\alpha \beta} h^{\alpha} \otimes h^{\beta}=\left(h_{\alpha} \cdot \frac{\partial t_{3}}{\partial X^{\beta}}-h_{\alpha}^{o} \cdot \frac{\partial t_{3}^{o}}{\partial X^{\beta}}\right) h^{\alpha} \otimes h^{\beta}, \\
\tau=\tau_{\alpha} h^{\alpha}=\left(t_{3} \cdot \frac{\partial r}{\partial X^{\alpha}}-t_{3}^{o} \cdot \frac{\partial r_{o}}{\partial X^{\alpha}}\right) h^{\alpha},
\end{gathered}
$$

where $\tau_{\alpha}, \rho_{\alpha \beta}$ and $\epsilon_{\alpha \beta}(\alpha, \beta=1,2)$ measure the transverse shearing, the curvature and the membrane stretching and shearing of the shell in the two material directions $X^{1}$ and $X^{2}$, with respect to the reference configuration (which is throughout the article distinguished with the upper index $o$ ). From section 2.4, we also have $\left(h_{1}, h_{2}\right)(\bar{X})=\left(\partial r / \partial X^{1}, \partial r / \partial X^{2}\right)(\bar{X})=\left(R . \Gamma_{1}, R \cdot \Gamma_{2}\right)(\bar{X})$, and $t_{3}=R \cdot E_{3}$, which once introduced into 83 85 give the following alternative expressions of the strain measures:

$$
\begin{gathered}
\epsilon_{\alpha \beta}=\frac{1}{2}\left(\Gamma_{\alpha} \cdot \Gamma_{\beta}-\Gamma_{\alpha}^{o} \cdot \Gamma_{\beta}^{o}\right), \\
\rho_{\alpha \beta}=E_{3} \cdot\left(K_{\alpha} \times \Gamma_{\beta}-K_{\alpha}^{o} \times \Gamma_{\beta}^{o}\right), \\
\tau_{\alpha}=E_{3} \cdot\left(\Gamma_{\alpha}-\Gamma_{\alpha}^{o}\right),
\end{gathered}
$$

which show that as expected, the strain measures of (83), (84), and 850 only depend on the left invariant fields $\xi_{\alpha}=\left(K_{\alpha}^{T}, \Gamma_{\alpha}^{T}\right)^{T}$ of $(66)$.

Constitutive equations for classical shells In the simplest case, where the shell is made of an isotropic hyperelastic material, we define a density of internal strain energy per unit of metric reference volume of $\left(\Phi_{o} \circ e\right)(\mathcal{D})$ in the form 
$\mathfrak{U}_{o}=\bar{\rho}_{o} \psi$, where $\psi$ is a point-wise function (on $\left.\mathcal{D}\right)$ of the strains $(86,88)$. Then, invoking the conservation of mass (67), or equivalently a relation similar to (46), but for strains $(86-88)$, the internal stress state can be defined by the properly invariant hyperelastic constitutive relations:

$$
\mathcal{N}_{t}^{\alpha \beta}=\bar{\rho}_{t} \frac{\partial \psi}{\partial \epsilon_{\alpha \beta}}, \mathcal{M}_{t}^{\alpha \beta}=\bar{\rho}_{t} \frac{\partial \psi}{\partial \rho_{\alpha \beta}}, \mathcal{Q}_{t}^{\alpha}=\bar{\rho}_{t} \frac{\partial \psi}{\partial \tau_{\alpha}},
$$

where $\mathcal{N}_{t}=\mathcal{N}_{t}^{\alpha \beta} h_{\alpha} \otimes h_{\beta}, \mathcal{M}_{t}=\mathcal{M}_{t}^{\alpha \beta} h_{\alpha} \otimes h_{\beta}$ and $\mathcal{Q}_{t}=\mathcal{Q}_{t}^{\alpha} h_{\alpha}$ define a set of stress tensors, called "effective" in [12, which are entirely defined as the dual of the strain fields $(83$ 85) according to the following expression of the virtual work of the internal forces exerted inside $\mathcal{B}$ :

$$
\delta W_{i n t}=\int_{\mathcal{D}}\left(\mathcal{N}_{t}^{\alpha \beta} \delta \epsilon_{\alpha \beta}+\mathcal{M}_{t}^{\alpha \beta} \delta \rho_{\alpha \beta}+\mathcal{Q}_{t}^{\alpha} \delta \tau_{\alpha}\right) \sqrt{|h|} d X^{1} \wedge d X^{2} .
$$

Defined in this way, the effective stress tensor $\mathcal{N}_{t}$ inherits of the symmetry (w.r.t. the $(\alpha, \beta)$ indices) of the strain measures $(86)$ and satisfies by construction $\mathcal{N}_{t}^{\alpha \beta}=\mathcal{N}_{t}^{\beta \alpha}(\alpha, \beta=1,2)$, a restriction which will be discussed further in next subsection. For the time being, note that the Cosserat stress density $\Lambda_{t}^{\alpha}=\left(M_{t}^{\alpha T}, N_{t}^{\alpha T}\right)^{T}$ of the Poincaré-Cosserat equations 75 are related to the effective stress through the relation:

$$
\Lambda_{t}^{\gamma}=\bar{\rho}_{t}\left(\frac{\partial \psi}{\partial \epsilon_{\alpha \beta}}\right)\left(\frac{\partial \epsilon_{\alpha \beta}}{\partial \xi_{\gamma}}\right)+\bar{\rho}_{t}\left(\frac{\partial \psi}{\partial \rho_{\alpha \beta}}\right)\left(\frac{\partial \rho_{\alpha \beta}}{\partial \xi_{\gamma}}\right)+\bar{\rho}_{t}\left(\frac{\partial \psi}{\partial \tau_{\alpha}}\right)\left(\frac{\partial \tau_{\alpha}}{\partial \xi_{\gamma}}\right),
$$

which can be detailed, by using the definitions of effective strains $86 \mid 88)$ and stress $(89)$, as:

$$
\Lambda_{t}^{\alpha}=\left(\begin{array}{c}
M_{t}^{\alpha} \\
N_{t}^{\alpha}
\end{array}\right)=\left(\begin{array}{c}
\mathcal{M}_{t}^{\alpha \beta}\left(E_{3} \times \Gamma_{\beta}\right) \\
\mathcal{M}_{t}^{\beta \alpha}\left(K_{\beta} \times E_{3}\right)+\mathcal{N}_{t}^{\alpha \beta} \Gamma_{\beta}+\mathcal{Q}_{t}^{\alpha} E_{3}
\end{array}\right) .
$$

Finally, by substituting $86-88$ into 89 and the result into $(92)$, we obtain a constitutive law for classical shells which relates the left invariant fields of (65) to their dual (69).

Role of the constitutive law in the reduction of the internal stress state The aim of this subsection is to show how the above constitutive law (89) completely takes charge the process of degenerating the micro-solid $\mathcal{M}$ into directors (i.e. one-dimensional rigid bodies) in the stress model. To achieve this reduction process, we can take inspiration from the reduction of the angular balance equations of a 3D Cosserat (micropolar) medium into those of a classical (non-Cosserat) one. Thus, we reconsider the model of full Cosserat shells 75 , in which we remove $M_{t}^{31}$ and $M_{t}^{32}$, along with the kinetic momenta $\Sigma_{t}^{3}=\left(\bar{j}_{\|} / \sqrt{h}\right) \Omega_{3}$ and external couples $M_{\text {ext, } t}^{3}$ around the directors $t_{3}$. This simplification changes the sixth row of 75 into:

$$
E_{3} \cdot\left(\Gamma_{\alpha} \times N_{t}^{\alpha}+\left(K_{\alpha} \times E_{3}\right) \times\left(M_{t}^{\alpha} \times E_{3}\right)\right)=0,
$$


which models the (degenerated) angular dynamics about the directors with no intrinsic spin and couple stress. Expression (93) stands for a static constitutive constraint on the internal stress of the classical shell model. Moreover, inserting (92) into (93) and achieving simple vector algebra allows changing (93) into the following constitutive constraint on the effective stress tensor components:

$$
\mathcal{N}_{t}^{\alpha \beta}\left(\Gamma_{\alpha} \times \Gamma_{\beta}\right) \cdot E_{3}=0,
$$

which obliges the effective stress tensor $\mathcal{N}_{t}=\mathcal{N}_{t}^{\alpha \beta} h_{\alpha} \otimes h_{\beta}$ to be symmetric, as the Cauchy stress tensor field is in a classical (not Cosserat) 3D medium 33 . Thus, the constitutive law $(89)$ imposing defacto the symmetry condition $(\overrightarrow{94})$, it implicitly forces the constitutive constraint (93), and the degenerated angular balance along the directors, from which it derives. Finally, the internal stress state dimension has been reduced from the 12 components of the Cosserat-stress tensor-densities $\left(M_{t}^{\alpha}, N_{t}^{\alpha}\right)$ to the 8 components of the effective stress fields $\mathcal{N}_{t}^{\alpha \beta}$, $\mathcal{M}_{t}^{\alpha \beta}$ and $\mathcal{Q}_{t}^{\alpha}$, if, as it is usually done, the skew symmetric components of the couple stress $\mathcal{M}_{t}=\mathcal{M}_{t}^{\alpha \beta} h_{\alpha} \otimes h_{\beta}$ are forced to zero by restricting the constitutive law $\left(89\right.$-midle) to the symmetric part of $\mathcal{M}_{t}$. For each dimension of $\mathcal{M}$ which is degenerated, $p$ couple stress components are removed. This first simplification forces a second one through the angular momentum balance which leads to the removal of the $\left(p^{2}-p\right) / 2=p(p-1) / 2$ symmetric components of the stress tensor. In the $3 \mathrm{D}$ case, $p=3$, and this process reduces the stress state from the 6. $p=18$ components of the Cauchy stress and couple stress tensors to the $18-3.3-3(3-1) / 2=6$ independent components of the Cauchy-stress tensor. In the $2 \mathrm{D}$ case, we have $p=2$ and the same process reduces the stress state from the 6. $p=12$ components of the full Cosserat model to $12-1.2-2(2-1) / 2=9$ and finally, with the couple stress tensor symmetry, to the 8 independent components of the effective stress tensors and vectors.

This reduction process is schematized in figure 4 . It leads to a closed formulation for classical shells which consists of the balance equations 80 or 81 with their boundary conditions, the reconstruction equation 82, the definition of the strains 83 85 and the constitutive law 89 , with 92 relating the Cosserat stress in 80) and (81) with the effective stress of 89.

\section{Geometrically exact model of classical shells with drilling rotations}

It is worth noting that in the above model of classical shell, the angular velocity around the directors, "named drilling rotation" in shell literature [27], is assumed to be zero, i.e, $\Omega_{3}=0$, in 80 , (81) and 82 . Though it has no consequence on the dynamics of the directors, this condition is kinematically inconsistent with the mid-surface shell deformation. In this section, we reintroduce this additional rotation at the kinematic stage 25 of the state-space formulation, and derive a closed formulation of classical shells with a model of drilling rotations consistent 


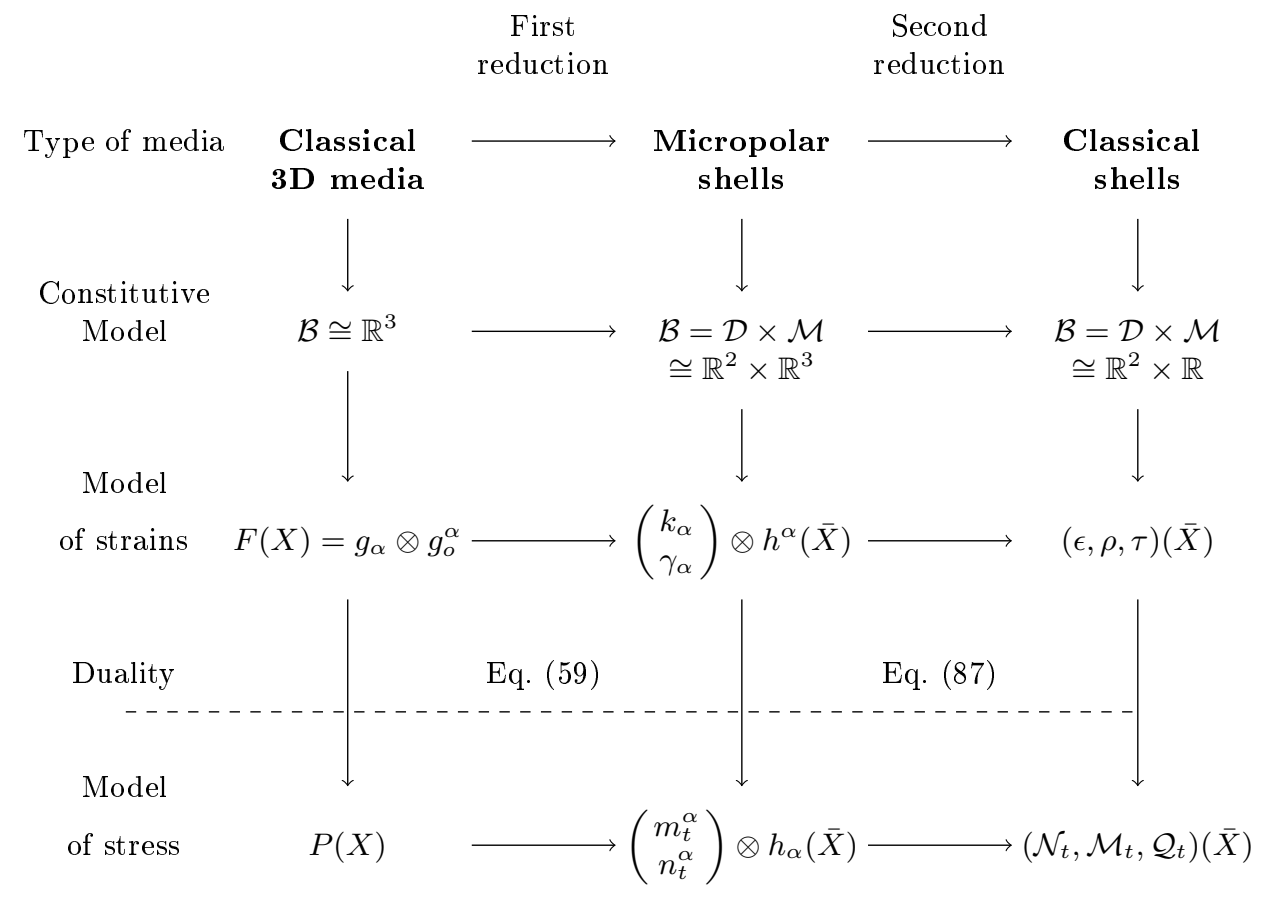

Fig. 4. Reduction process for classical shells. The first reduction is based on Cosserat kinematics (8) and leads to the micropolar model of section 6 , the second is based on the elimination of couple stress and intrinsic spin about directors, along with the constitutive law 89p. It leads to the models of classical shells of section 7 and 8 . 
with the mid-surface deformation. To that end, we fixe the rotation around the directors, to ensure that the full field $R$ matches the rotational part of the polar decomposition of the gradient of the transformation (64) restricted to the midsurface shell. The restricted gradient has been defined as $\nabla \bar{\Phi}=h_{\alpha} \otimes E^{\alpha}$ (see section 2.4). Inspired by [40], this approach has been originally applied to the restricted gradient of transformations $\bar{F} \triangleq h_{\alpha} \otimes h_{o}^{\alpha}$ in the context of the finite element method for geometrically exact shells $[28$. In our context, it consists of fixing the drilling rotation about the directors at each instant, by imposing the symmetry of the tensor $\left(R^{T} . \nabla \bar{\Phi}\right)(\bar{X})=\bar{U}(\bar{X})$, where $\bar{U}$ is a symmetric tensor on the shell mid-surface measuring the stretching and shearing between the material mid surface and its deformed configuration. As a result, imposing the symmetry of $\bar{U}$ on the mid-surface is equivalent to force for $\alpha, \beta=1,2$ :

$$
E_{\alpha} \cdot \bar{U} \cdot E_{\beta}=E_{\beta} \cdot \bar{U} \cdot E_{\alpha} .
$$

But since we also have:

$$
E_{\alpha} \cdot\left(R^{T} \cdot \nabla \bar{\Phi}\right) \cdot E_{\beta}=E_{\alpha} \cdot\left(R^{T} \cdot\left(h_{\gamma} \otimes E^{\gamma}\right)\right) \cdot E_{\beta}=E_{\alpha} \cdot\left(R^{T} \cdot h_{\beta}\right)=E_{\alpha} \cdot \Gamma_{\beta},
$$

the dynamically undeterminate drilling rotation is fixed by forcing the following point-wise geometric constraint on the shell mid-surface:

$$
E_{1} \cdot \Gamma_{2}=E_{2} \cdot \Gamma_{1} .
$$

Time-differentiating (97) gives the following expression of the missing angular field, with $\operatorname{Tr}(K)=K_{11}+K_{22}$ and summations on $\alpha$ :

$$
\Omega_{3}=\frac{1}{\Gamma_{11}+\Gamma_{22}}\left[\left(\frac{\partial V_{2}}{\partial X^{1}}-\frac{\partial V_{1}}{\partial X^{2}}\right)-\operatorname{Tr}(K) V_{3}+K_{3 \alpha} V_{\alpha}+\Gamma_{3 \alpha} \Omega_{\alpha}\right] .
$$

It is worth noting here that $(98)$ is the shell counterpart of the angular velocity field defined as the skew-symmetric part of the 3D velocity gradient in the classical (not Cosserat) 3D media. As an illustration, consider the case of a rigid plate, where $K_{\alpha}=0, \Gamma_{3 \alpha}=0$, and $\Gamma_{11}=\Gamma_{22}=1$ are imposed in (98). As expected, $\Omega_{3}=(1 / 2)\left(\partial V_{2} / \partial X^{1}-\partial V_{1} / \partial X^{2}\right)$, i.e., $\Omega_{3}$ is equal to the half curl of the linear velocity field on the rigid plate mid-surface. Finally, once inserted in (78), the kinematic model (98) of $\Omega_{3}$ completes the degenerated dynamics which now govern the time evolution of $V$ and $\Omega_{\alpha}, \alpha=1,2$. It is worth noting here that as this model of the drilling rotation is only kinematic, and not dynamic, the reference configuration plays no role other than fixing the initial conditions of the state-space equations through the field of frame $\bar{X} \mapsto R_{o}(\bar{X})$, which support the directors at $t=0$. In particular, the symmetric tensor $\bar{U}$ of the above construction does not match with the stretching tensor related to the mechanical states in the reference and deformed configuration.

To conclude this section, a closed formulation for classical shells with a consistent model of drilling rotations consists of the strains (83,85), the constitutive law 89 ) and (92), along with the balance equations 80) (or their counterparts 


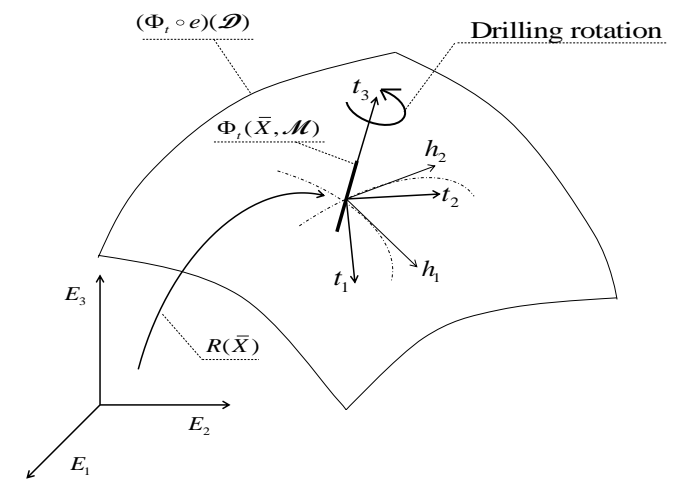

Fig. 5. Sketch of the drilling rotation in classical Cosserat shells.

related to the reference configuration), and the reconstruction equation (78), where in (78), $\Omega_{3}$ is given by (98).

\section{Illustrative example: application to axisymmetric shells}

In this section we illustrate the previous general picture by applying it to an axisymmetric shell $\mathcal{B}$ with a mid-surface diffeomorphic to a disc as pictured in figure 6 . The shell undergoes a net translation and axisymmetric shape deformations along the $\left(o, e_{3}\right)$ direction of an inertial frame $\left(o, e_{1}, e_{2}, e_{3}\right)$ with no rotation around it. Thus, there is no couple nor rotation exerted around the micro-structures and the full Cosserat model can be applied even if the shell is classical. According to the problem symmetry, the inertial frame $\left(o, e_{1}, e_{2}, e_{3}\right)$ is endowed with a chart of cylindrical coordinates $(r, \phi, z)$ of local orthonormal basis $\left(e_{r}, e_{\phi}, e_{3}\right)$. The material space $\mathcal{B}$, of material frame $\left(O, E_{1}, E_{2}, E_{3}\right)=$ $\left(o, e_{1}, e_{2}, e_{3}\right)$, is identified to $\mathcal{D} \times \mathcal{M}$, with $\mathcal{D}$ the shell's material mid surface supported by $\left(E_{1}, E_{2}\right)$, and $\mathcal{M}$ its director supported by $E_{3}$, and crossed by $\mathcal{D}$, in its center ( $l$ and $j$ denote the length and the perpendicular angular geometric inertia moment of $\mathcal{M})$. The reference configuration is $\Phi_{o}(\mathcal{B})$. Its symmetry axis is $\left(O, e_{3}\right)$, and the cylindrical coordinates of its points are denoted $\left(r_{o}, z_{o}, \phi_{o}\right)$. In this context, the open set $\mathcal{D}-\{\partial \mathcal{D} \cup O\}$ is covered with the material chart $\left\{X^{1}, X^{2}\right\}=\{X, \phi\}$ of natural basis $\left\{E_{1}, E_{2}\right\}=\{\partial / \partial X, \partial / \partial \phi\}$, where $X$ is the metric length along the meridians of $\left(\Phi_{o} \circ e\right)(\mathcal{D})$. In any configuration $\Phi(\mathcal{B})$ of the shell, any cross section fiber crossing $\mathcal{D}$ in $(X, \phi)$, is supported by the third unit vector of a director frame $\left(t_{1}, t_{2}, t_{3}\right)(X, \phi)$ deduced from $\left(O, E_{1}, E_{2}, E_{3}\right)$ through a transformation of $S E(3)$ of the form:

$$
\mathrm{g}(X, \phi)=\left(\begin{array}{cc}
\exp \left(\phi \hat{e}_{3}\right) & 0 \\
0 & 1
\end{array}\right)\left(\begin{array}{cc}
\exp \left(-\theta \hat{e}_{\phi}\right) r e_{r}+z e_{3} \\
0 & 1
\end{array}\right)
$$




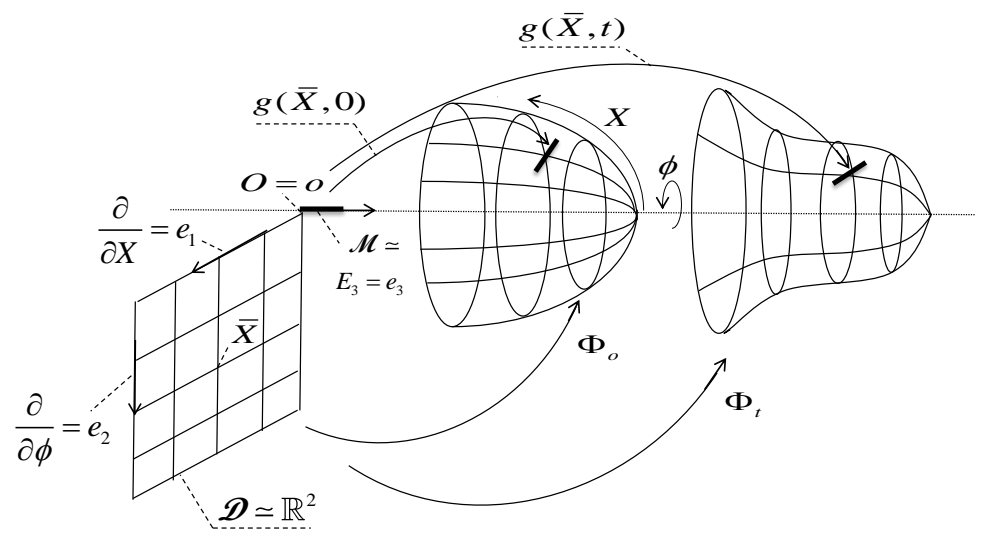

Fig. 6. Application of the construction to an axisymmetric shell.

with $\theta$, the angle parameterizing the local rotation of the director $t_{3}$ with respect to $e_{3}$. Using $(99)$ in $(65)$, gives with $s e(3) \cong \mathbb{R}^{6}$, the three left invariant fields:

$\eta=\left(\begin{array}{c}0 \\ \Omega_{2} \\ 0 \\ V_{1} \\ 0 \\ V_{3}\end{array}\right), \xi_{X}=\left(\begin{array}{c}0 \\ K_{2 X} \\ 0 \\ \Gamma_{1 X} \\ 0 \\ \Gamma_{3 X}\end{array}\right)=\left(\begin{array}{c}0 \\ -\theta^{\prime} \\ 0 \\ r^{\prime} \cos \theta+z^{\prime} \sin \theta \\ 0 \\ z^{\prime} \cos \theta-r^{\prime} \sin \theta\end{array}\right), \xi_{\phi}=\left(\begin{array}{c}K_{1 \phi} \\ 0 \\ K_{3 \phi} \\ 0 \\ \Gamma_{2 \phi} \\ 0\end{array}\right)=\left(\begin{array}{c}\sin \theta \\ 0 \\ \cos \theta \\ 0 \\ r \\ 0\end{array}\right)$,

where' denotes $\partial . / \partial X$. These expressions define $\Gamma_{\alpha}$ and $K_{\alpha}(\alpha=X, \phi)$, which once inserted in (86 88), give the expressions of (effective) strain components:

$$
\begin{aligned}
& \left(\begin{array}{c}
\tau_{X} \\
\tau_{\phi}
\end{array}\right)=\left(\begin{array}{c}
\Gamma_{3 X} \\
0
\end{array}\right),\left(\begin{array}{cc}
\epsilon_{X X} & \epsilon_{X \phi} \\
\epsilon_{\phi X} & \epsilon_{\phi \phi}
\end{array}\right)=\left(\begin{array}{cc}
\Gamma_{1 X}^{2}+\Gamma_{3 X}^{2}-1 & 0 \\
0 & \Gamma_{2 \phi}^{2}-r_{o}^{2}
\end{array}\right), \\
& \left(\begin{array}{cc}
\rho_{X X} & \rho_{X \phi} \\
\rho_{\phi X} & \rho_{\phi \phi}
\end{array}\right)=\left(\begin{array}{cc}
K_{2 X}^{o} \Gamma_{1 X}^{o}-K_{2 X} \Gamma_{1 X} & 0 \\
0 & K_{1 \phi} \Gamma_{2 \phi}-r_{o} K_{1 \phi}^{o}
\end{array}\right),
\end{aligned}
$$

where we assume no transverse shearing in the reference configuration. Then, introducing the Cosserat and effective stress of the general construction, and remarking that the axisymmetry imposes: $\mathcal{N}_{t}^{X \phi}=\mathcal{N}_{t}^{\phi X}=0, \mathcal{M}_{t}^{X \phi}=\mathcal{M}_{t}^{\phi X}=0$ 
and $\mathcal{Q}_{t}^{\phi}=0,92$ allows writing the relations between the Cosserat and the effective stress tensors components, all related to the reference configuration, as:

$$
\left(\begin{array}{c}
0 \\
M_{o}^{2 X} \\
0 \\
N_{o}^{1 X} \\
0 \\
N_{o}^{3 X}
\end{array}\right)=\left(\begin{array}{c}
0 \\
\Gamma_{1 X} \mathcal{M}_{o}^{X X} \\
0 \\
K_{1 \phi} \mathcal{M}_{o}^{X X}+\Gamma_{1 X} \mathcal{N}_{o}^{X X} \\
0 \\
\Gamma_{3 X} \mathcal{N}_{o}^{X X}+\mathcal{Q}_{o}^{X}
\end{array}\right),\left(\begin{array}{c}
M_{o}^{1 \phi} \\
0 \\
0 \\
0 \\
N_{o}^{2 \phi} \\
0
\end{array}\right)=\left(\begin{array}{c}
-\Gamma_{2 \phi} \mathcal{M}_{o}^{\phi \phi} \\
0 \\
0 \\
0 \\
\Gamma_{2 \phi} \mathcal{N}_{o}^{\phi \phi}-K_{2 X} \mathcal{M}_{o}^{\phi \phi} \\
0
\end{array}\right) .
$$

Applying the material Poincare-Cosserat equations 75 related to the reference configuration $\left(\Phi_{o} \circ e\right)(\mathcal{D})$ to our shell, with the expressions of $\eta, \xi_{X}, \xi_{\phi}$ above, $\sqrt{\left|h_{o}\right|}=\left(\Gamma_{2 \phi}^{o}\left(\Gamma_{1 X}^{o}\right)^{2}+\left(\Gamma_{3 X}^{o}\right)^{2}\right)^{1 / 2}=r_{o}$, and no dependency of the Lagrangian density with respect to $\phi$, gives the three following scalar equations :

$$
\begin{aligned}
& \rho_{o} l\left(\frac{\partial V_{1}}{\partial t}-V_{3} \Omega_{2}\right)=\frac{1}{r_{o}} \frac{\partial r_{o} N_{o}^{1 X}}{\partial X}+K_{2 X} N_{o}^{3 X}-K_{3 \phi} N_{o}^{2 \phi}+N_{e x t, o}^{1}, \\
& \rho_{o} l\left(\frac{\partial V_{2}}{\partial t}+V_{1} \Omega_{2}\right)=\frac{1}{r_{o}} \frac{\partial r_{o} N_{o}^{3 X}}{\partial X}-K_{2 X} N_{o}^{1 X}+K_{1 \phi} N_{o}^{2 \phi}+N_{e x t, o}^{2}, \\
& \rho_{o} j \frac{\partial \Omega_{2}}{\partial t}=\frac{1}{r_{o}} \frac{\partial r_{o} M_{o}^{2 X}}{\partial X}-\Gamma_{1 X} N_{o}^{3 X}+\Gamma_{3 X} N_{o}^{1 X}+K_{3 \phi} M_{o}^{1 \phi}+M_{e x t, o}^{2} .
\end{aligned}
$$

Pushing forward these equations in the field of mobile director frames $(X, \phi) \mapsto$ $\left(t_{1}, t_{2}, t_{3}\right)(X, \phi)$ with $t_{i}(X, \phi)=R(X, \phi) . E_{i}$ and remarking that $\partial t_{2} / \partial \phi=-e_{r}$, gives two equations (one vectorial and one scalar) which represent the spatial Poincaré equations (77) for an axisymmetric shell:

$$
\begin{aligned}
& \rho_{o} r_{o} l\left(\frac{\partial v}{\partial t}\right)=\frac{\partial}{\partial X}\left[r_{o}\left(N_{o}^{1 X} t_{1}+N_{o}^{3 X} t_{3}\right)\right]-r_{o}\left(N_{o}^{2 \phi} e_{r}-n_{e x t, o}\right), \\
& \rho_{o} r_{o} j\left(\frac{\partial \Omega_{2}}{\partial t}\right)=\frac{\partial}{\partial X}\left[r_{o} M_{o}^{2 X}\right]+r_{o}\left(K_{3 \phi} M_{o}^{1 \phi}+\Gamma_{3 X} N_{o}^{1 X}-\Gamma_{1 X} N_{o}^{3 X}+M_{e x t, o}^{2}\right),
\end{aligned}
$$

where $v=R . V$. Moreover, introducing the densities of internal wrench per unit of metric length of the material coordinate lines on the reference configuration as defined in section 6.4, that we distinguish from the same densities, but per unit of material length, by a star $\star$, we find in the present case, i.e., with $\left|h_{o, X X}\right|=1$, $\left|h_{o, \phi \phi}\right|=r_{o}^{2}$ :

$$
M_{o}^{X}=\left(r_{o} / r_{o}\right) M_{o}^{\star X}, N_{o}^{X}=\left(r_{o} / r_{o}\right) N_{o}^{\star X}, M_{o}^{\phi}=\left(1 / r_{o}\right) M_{o}^{\star \phi}, N_{o}^{\phi}=\left(1 / r_{o}\right) N_{o}^{\star \phi} .
$$

Once inserted in (101), these relations give an alternative form of balance equations in terms of metric densities of stress:

$$
\begin{aligned}
& \rho_{o} r_{o} l \frac{\partial v}{\partial t}=\frac{\partial}{\partial X}\left[r_{o}\left(N_{o}^{\star 1 X} t_{1}+N_{o}^{\star 3 X} t_{3}\right)\right]-N_{o}^{\star 2 \phi} e_{r}+r_{o} n_{e x t, o}, \\
& \rho_{o} r_{o} j \frac{\partial \Omega_{2}}{\partial t}=\frac{\partial}{\partial X}\left[r_{o} M_{o}^{\star 2 X}\right]+K_{3 \phi} M_{o}^{\star 1 \phi}+r_{o}\left(\Gamma_{3 X} N_{o}^{\star 1 X}-\Gamma_{1 X} N_{o}^{\star 3 X}+M_{e x t, o}^{2}\right),
\end{aligned}
$$


which are the classical equations of axisymmetric shells as given in [25], where they are expressed in terms of director stress couples. These balance equations, which involve densities of wrenches per unit of metric volume of $\left(\Phi_{o} \circ e\right)(\mathcal{D})$, have to be supplemented with the expressions of $\eta, \xi_{X}$ and $\xi_{\phi}$, the constitutive equations in terms of effective stresses (89), the reconstruction equation (78) with their initial conditions, and a model of external forces $\left(N_{e x t, o}^{1}, N_{e x t, o}^{2}, M_{e x t, o}^{2}\right)$.

\section{Conclusion and perspectives for soft robotics}

In this article we proposed a general picture which allowed the partial differential equations and the boundary conditions of a Cosserat medium, i.e., a set of small rigid bodies continuously staked along one or several material dimensions, to be derived. This is achieved through a variational calculus from a unique Lagrangian density and its symmetry group. The approach is systematic and requires no phenomenological input. It is based on an extension of the Poincaré equations from classical mechanical systems to field theory. As with the classical Poincaré equations, these equations are stated in the dual of the Lie algebra of the symmetry group of the Lagrangian. As a result, they are a set of first order dynamics equations governing the medium's velocities, i.e., the components of the infinitesimal right (material) or left (spatial) transformations of the group's Lie algebra. When the system is fully symmetric, they can be time-integrated autonomously in a first step, and in a second step, the resulting velocity field allows the recovery of the motion of the medium's transformations on the group, through reconstruction equations. Remarkably, these general equations allow the recovery of the usual formulations of non-linear shells theories in both the reference and current configuration, and in the material and spatial setting. More precisely, this may be achieved from a unique set of covariant partial differential equations leading to all the formulations deduced from each other through some transformations which change densities related to the material, reference and deformed configurations, and others which change material into spatial infinitesimal transformations. While the approach gives a fully determinate dynamic model of micro-polar shells, it has been reduced further to obtain a model of classical shells with no couple stress, nor spin about their directors. This reduction process is based on the use of constitutive laws imposing the symmetry of a set of effective stress defined in the shell's mid surface. Due to the indeterminacy of the drilling rotation about the directors, the shell motion reconstruction requires a kinematic model of the missing rotation deduced from the polar decomposition of the gradient of the mid-surface transformation.

In future, this work will be used as foundations to address some open issues in the emerging field of underwater soft robotics 22 . Just like the beam PoincaréCosserat equations have been proved to be a powerful modelling tool for fish swimming in biorobotics [41, 42, their extension to shells can greatly help in modelling squids. In fact, these animals have evolved a soft open shell cavity, a mantle, that they cyclically contract to perform jet propulsion, which is an 
emerging research topic in bio-fluid dynamics 43 . Modelling the mantle as an internally actuated axisymmetric Cosserat shell immersed in a quiescent fluid, the above Poincaré-Cosserat abstract machinery will be of great help to generate the squid swimming dynamics equations in a blind manner from a minimum set of physical inputs, here fed into a Lagrangian. In this perspective, let us remark that beyond the modeling of body inertial forces addressed in the article, the reactive hydrodynamic forces that are exerted on the body can be modelled by adding some fluid contributions to the Lagrangian of the body, according to the Kirchhoff potential approach [44]. On the other hand, the body strain energy density can be used to help in modelling the animal muscles and their technological emulations (cables, electro-active materials...). Of course, to achieve our final goal, several evolutions of this picture are already expected. In particular, starting from the Eulerian realm of fluid mechanics with no model of the boundary layer, the Kirchhoff model of hydrodynamic forces will need to be supplemented with a simplified model of the vorticity, while the fluid kinetic energy will need to extend the above picture to systems (body + fluid) enjoying weaker (global) symmetries. Finally, the above picture encompassing both beams and shells, it can help to build a theory for soft-multibody systems with applications to octopus.

\section{Appendix 1: Proof of (44) through direct application of the Hamilton principle}

As evoked in remark 5.2, equations (43) and (44) can be derived directly by applying the Hamilton principle to an action defined in terms of the different definitions of the reduced Lagrangian of section 2.6. In this Appendix, we apply this calculus to the deformed configuration. This can be achieved using Stokes theorem applied to differential forms or alternatively using the divergence theorem and vector analysis. We will follow the latter approach and will denote, according to the context of section $2.4, d X^{1} d X^{2} \ldots d X^{p}=d \mathcal{D}$ and $|\bar{H}|^{1 / 2} d Y^{1} d Y^{2} \ldots d Y^{p-1}=d \partial \mathcal{D}$ the material volumes of $\mathcal{D}$ and $\partial \mathcal{D}$, which are assumed to be two manifolds consistently oriented according to the outward unit normal convention. We start from $\sqrt{19}$ and $(20)$ in which we replace $\mathfrak{L}$ by $\sqrt{|h|} \mathfrak{L}_{t}$ and $\left(F_{\text {ext }}, \bar{F}_{\text {ext }}\right)$ by $\left(F_{\text {ext }}|h|^{1 / 2}, F_{\text {ext }}|\bar{h}|^{1 / 2}\right)$. In this new formulation of Hamilton principle, $\delta$ being achieved at fixed time and material parameters according to section 2.6, this enables us to shift it under the integral. Then, since $\delta\left(\mathfrak{L}_{t} \sqrt{|h|}\right)=\delta \mathfrak{L}_{t} \sqrt{|h|}+\mathfrak{L}_{t} \delta \sqrt{|h|}$, where $\sqrt{|h|}$ is configuration-dependent through the invariant fields $\xi_{\alpha}$ (see section 2.6), we have:

$$
\int_{t_{1}}^{t_{2}} \int_{\mathcal{D}}\left(<\frac{\partial \mathfrak{L}_{t}}{\partial \eta}, \delta \eta>+<\left[\frac{\partial \mathfrak{L}}{\partial \xi_{\alpha}}\right]_{t}, \delta \xi_{\alpha}>\right) \sqrt{|h|} d \mathcal{D} d t=-\int_{t_{1}}^{t_{2}} \delta W_{\text {ext }} d t
$$

where we used the notation $\left[\partial \mathfrak{L} / \partial \xi_{\alpha}\right]_{t}=\partial \mathfrak{L}_{t} / \partial \xi_{\alpha}+|h|^{-\frac{1}{2}}\left(\partial|h|^{\frac{1}{2}} / \partial \xi_{\alpha}\right) \mathfrak{L}_{t}$, as it is introduced by (42). Then invoking (23) and applying a by-part time-integration with fixed extreme times condition, allows the left hand side of 102 to be 
rewritten as:

$$
\begin{aligned}
& \int_{t_{1}}^{t_{2}} \int_{\mathcal{D}}<\frac{1}{\sqrt{|h|}} a d_{\eta}^{*}\left(\sqrt{|h|} \frac{\partial \mathfrak{L}_{t}}{\partial \eta}\right)-\frac{1}{\sqrt{|h|}} \frac{\partial}{\partial t}\left(\sqrt{|h|} \frac{\partial \mathfrak{L}_{t}}{\partial \eta}\right), \delta \zeta>\sqrt{|h|} d \mathcal{D} d t \\
& +\int_{t_{1}}^{t_{2}} \int_{\mathcal{D}}<\left[\frac{\partial \mathfrak{L}}{\partial \xi_{\alpha}}\right]_{t}, \frac{\partial \delta \zeta}{\partial X^{\alpha}}+a d_{\xi_{\alpha}}(\delta \zeta)>\sqrt{|h|} d \mathcal{D} d t .
\end{aligned}
$$

Now let us remark that:

$$
\begin{aligned}
& \int_{\mathcal{D}}<\left[\frac{\partial \mathfrak{L}}{\partial \xi_{\alpha}}\right]_{t}, \frac{\partial \delta \zeta}{\partial X^{\alpha}}>\sqrt{|h|} d \mathcal{D}= \\
& \int_{\mathcal{D}} \frac{\partial}{\partial X^{\alpha}}\left(\sqrt{|h|}<\left[\frac{\partial \mathfrak{L}}{\partial \xi_{\alpha}}\right]_{t}, \delta \zeta>\right) d \mathcal{D}-\int_{\mathcal{D}}<\frac{\partial}{\partial X^{\alpha}}\left(\sqrt{|h|}\left[\frac{\partial \mathfrak{L}}{\partial \xi_{\alpha}}\right]_{t}\right), \delta \zeta>d \mathcal{D}
\end{aligned}
$$

whose the first right-hand-side term is merely the divergence of a vector field of contravariant components $v^{\alpha}=<\left[\partial \mathfrak{L} / \partial \xi_{\alpha}\right]_{t}, \delta \zeta>$ in the convected basis $\left\{h_{\alpha}\right\}_{\alpha=1, . . p}$. Applying the divergence theorem to this term gives:

$$
\int_{\mathcal{D}} \frac{\partial}{\partial X^{\alpha}}\left(\sqrt{|h|}<\left[\frac{\partial \mathfrak{L}}{\partial \xi_{\alpha}}\right]_{t}, \delta \zeta>\right) d \mathcal{D}=\int_{\partial \mathcal{D}}<\left[\frac{\partial \mathfrak{L}}{\partial \xi_{\alpha}}\right]_{t}, \delta \zeta>\nu_{t, \alpha}|\bar{h}|^{1 / 2} d \partial \mathcal{D}
$$

where we introduce the metric volume element $|\bar{h}|^{1 / 2} d \partial \mathcal{D}$ on $\left(\Phi_{t} \circ e\right)(\partial \mathcal{D})$, and $\nu_{t, \alpha} h^{\alpha}$ is the unit outward normal to the tangent planes of $\left(\Phi_{t} \circ e\right)(\partial \mathcal{D})$ which, from $(12)$, is related to the outward unit normal $\nu_{\alpha} E^{\alpha}$ by $\nu_{t, \alpha}|\bar{h}|^{1 / 2} d \partial \mathcal{D}=$ $\nu_{\alpha} \sqrt{|h|} d \partial \mathcal{D}$. Then, inserting $(105)$ into 104 and the result into (103) whose the last term is dualized, gives, with (20), a balance of two integral components, one over $\mathcal{D}$ with metric volume $\sqrt{|h| d \mathcal{D}}$ and the second over $\partial \mathcal{D}$, whose metric volume is $|\bar{h}|^{1 / 2} d \partial \mathcal{D}$. This balance being satisfied for any variation $\delta \zeta \in \mathfrak{g}$, it gives the set of equations $(44)$, where due to $\left(21,|\bar{h}|^{1 / 2}=1\right.$ in the case of beams.

\section{Appendix 2: Proof of 62}

The general expression 106 is stated in [14] in the case of geometrically exact beams and plates, in this Appendix we prove it for shells. Since the medium $\mathcal{B}$ is classical (not micropolar), we have:

$$
\delta W_{\text {int }}=\int_{\mathcal{B}} P: \delta F \sqrt{\left|g_{o}\right|} d \mathcal{B}=\frac{1}{2} \int_{\mathcal{B}} \sigma^{i j} \delta g_{i j} \sqrt{|g|} d \mathcal{B}
$$

where $g_{i j}\left(g^{i} \otimes g^{j}\right)$ is the fundamental metric tensor in the convected basis of $\Phi_{t}(\mathcal{B})$, with determinant $|g|$, and $\sigma^{i j}\left(g_{i} \otimes g_{j}\right)$ is the Cauchy stress tensor in the same basis. Then introducing the Cosserat shell kinematics (64) into $g_{i j}=g_{i} . g_{j}$ with $g_{i}=\partial \Phi_{t} / \partial X^{i}$ gives:

$$
g_{\alpha \beta} \simeq \Gamma_{\alpha} \cdot \Gamma_{\beta}+E_{3} \cdot\left(\Gamma_{\alpha} \times K_{\beta}+\Gamma_{\beta} \times K_{\alpha}\right) X^{3}, g_{\alpha 3}=\Gamma_{\alpha} \cdot E_{3}, g_{33}=1,
$$


where, consistently with the first-order Cosserat kinematics (8), the $X^{3}$-quadratic terms are neglected in the expression of $g_{\alpha \beta}$. In these conditions, we recognize in (107) the time-dependent components of the strain measures 86 88). Now, since expressions (107) depend on the medium configuration through the left invariant fields $\Gamma_{\alpha}$ and $K_{\alpha}$ of 65 only, one can state:

$$
\delta g_{i j}=\left(\frac{\partial g_{i j}}{\partial \Gamma_{\alpha}}\right) \delta \Gamma_{\alpha}+\left(\frac{\partial g_{i j}}{\partial K_{\alpha}}\right) \delta K_{\alpha}
$$

in which, the partial derivatives depend on the mid-surface coordinates through the left invariant fields, and on $X^{3}$, in a linear way. Then, introducing (108) into (106), and integrating the result along the $X^{3}$-thickness dimension, gives a general expression of the virtual work of internal forces similar to $(62)$, along with the explicit expressions of the resultant of stress and couple stress $N_{t}^{\alpha}$ and $M_{t}^{\alpha}$ in terms of the three-dimensional Cauchy stress tensor:

$$
M_{t}^{\alpha}=\frac{1}{\sqrt{|h|}} \int_{\mathcal{M}}\left(\sigma^{i j} \frac{\partial g_{i j}}{\partial K_{\alpha}} \sqrt{|g|}\right) d X^{3}, N_{t}^{\alpha}=\frac{1}{\sqrt{|h|}} \int_{\mathcal{M}}\left(\sigma^{i j} \frac{\partial g_{i j}}{\partial \Gamma_{\alpha}} \sqrt{|g|}\right) d X^{3}
$$

where $i<j$.

\section{References}

1. A. C. Eringen. Microcontinuum field theories I: foundations and solids. Springer New York, 1998.

2. H. Poincaré. Sur une forme nouvelle des équations de la mécanique. Compte Rendu de l'Académie des Sciences de Paris, 132:369-371, 1901.

3. J. E. Marsden, R. Montgomery, and T. S. Ratiu. Reduction, symmetry, and phases in mechanics. Memoire AMS, 436, 1990.

4. C.-M Marle. On Henri Poincaré's note: "sur une forme nouvelle des equations de la mécanique". Journal of Geometry and Symmetry in Physics, 29:1-38, 2013.

5. D. G. Ebin and J.E. Marsden. Groups of diffeomorphism and the motion of an incompressible fluid. Ann. Math, 92:102-163, 1970.

6. V. I. Arnold. Sur la geometrie differentielle des groupes de Lie de dimension infinie et ses applications a l'hydrodynamique des fluides parfaits. Ann. Inst. J. Fourier, 16(1):319-361, 1966.

7. E. Cosserat and F. Cosserat. Théorie des corps déformables. Hermann, Paris, 1909.

8. J. F. Pommaret. Partial differential Equations and group theory. Kluwer Academic Publishers, first edition, 1994.

9. M. Castrillón López, T. S. Ratiu, and S. Shkoller. Reduction in principal fiber bundles: covariant Euler-Poincaré equations. Proc. Amer. Math. Soc., 128(7):2155$2164,2000$.

10. D. C. Spencer. Overdetermined systems of partial differential equations. Bull. Amer. Math. Soc., 75:1-114, 1965.

11. F. Boyer and D. Primault. The Poincaré-Chetayev equations and flexible multibody systems. Journal of Applied Mathematics and Mechanics, 69(6):925-942. http://hal.archives-ouvertes.fr/hal-00672477, 2005. 
12. J. C. Simo and D. D. Fox. On a stress resultant geometrically exact shell model. Part I: formulation and optimal parametrization, volume 72. Mar. 1989.

13. J. C. Simo and L. Vu-Quoc. On the dynamics in space of rods undergoing large motions - A geometrically exact approach. Computer Methods in Applied Mechanics and Engineering, 66(2):125 - 161, 1988.

14. J.C. Simo, J. E. Marsden, and P. S. Krishnaprasad. The hamiltonian structure of nonlinear elasticity: The material and convective representations of solids, rods, and plates. Archive of Rational Mechanics and Analysis, 104:125-183, 1988.

15. F. Gay-Balmaz, D. D. Holm, and T. S. Ratiu. Variational principles for spin systems and the kirchhoff rod. The Journal of Geometric Mechanics, 1(4):417$444,2009$.

16. F. Demoures, F. Gay-Balmaz, M. Kobilarov, and T. S. Ratiu. Multisymplectic lie group variational integrator for a geometrically exact beam in r3. Communications in Nonlinear Science and Numerical Simulation, 19(10):3492-3512, 2014.

17. D.D. Holm and V. Putkaradze. Nonlocal orientation-dependent dynamics of charged strands and ribbons. C. R. Acad. Sci. Paris, Ser. I 347:1093-1098, 2009.

18. D. C. P. Ellis, F. Gay-Balmaz, D. D. Holm, V. Putkaradze, and T. S. Ratiu. Symmetry reduced dynamics of charged molecular strands. Archive of Rational Mechanics and Analysis, 197(3):811-902, 2010.

19. F. Boyer, M. Porez, and W. Khalil. Macro-continuous computed torque algorithm for a three-dimensional eel-like robot. IEEE Transactions on Robotics, 22(4):763775, Aug. 2006.

20. F. Boyer, S. Ali, and M. Porez. Macro-continuous dynamics for hyper-redundant robots: Application to kinematic locomotion bio-inspired by elongated body animals. IEEE Transactions on Robotics, 28(2):303 - 317, April 2012.

21. F. Renda, M. Giorelli, M. Calisti, M. Cianchetti, and C. Laschi. Dynamic model of a multibending soft robot arm driven by cables. IEEE Transactions on Robotics, 30(5):1109-1122, 2014.

22. A. Verl, A. Albu-Schaeffer, and Oliver Brock (Editor). Soft Robotics: Transferring Theory to Application. Springer-Verlag, New York, 2015.

23. E. Reissner. The effect of transverse shear deformation on the bending of elastic plates. J. Appl. Mech., 12:69-76, 1945.

24. A. E. Green and P. M. Naghdi. Non-isothermal theory of rods, plates and shells. Int. Journal of Solids and Structures, 6:209-244, 1970.

25. S. S. Antman. Nonlinear Problems of Elasticity (Mathematical Sciences vol 107). New York: Springer, 2nd edition, 2055.

26. A. Libai and J. G. Simmonds. The Nonlinear Theory of Elastic Shells, 2nd edn. Cambridge University Press, Cambridge, 1998.

27. Thomas J.R. Hughes and F. Brezzi. On drilling degrees of freedom. Computer Methods in Applied Mechanics and Engineering, 72:105-121, 1989.

28. D. D. Fox and J. C. Simo. A drill rotation formulation for geometrically exact shells. Computer Methods in Applied Mechanics and Engineering, 98:329-343, 1992.

29. J. E. Marsden and T. J. R. Hughes. Mathematical Foundations of Elasticity. Dover edition, first edition, 1994.

30. L. Vu-Quoc. On the algebra of two point tensors and their applications. ZAMM. Z. angew. Math. Mech., 76(9):540-541, 1996.

31. V. I. Arnold. Mathematical methods in classical mechanics. Springer-Verlag, NewYork, 2nd edition, 1988.

32. A. Lichnerowicz. Elements de calcul tensoriel. Jacques Gabay, Paris, 1987.

33. L. E. Malvern. Introduction to the mechanics of a continuous medium. PrenticeHall, New-Jersey, 1969. 
34. D. D. Holm, J. E. Marsden, and T. S. Ratiu. The Euler-Poincaré equations and semidirect products with applications to continuum theories. Adv. In Math., 137:181, 1998.

35. J. E. Marsden and T. S. Ratiu. Introduction to Mechanics and Symmetry. SpringerVerlag, 2nd edition, 1999.

36. A. E. Green and W. Zerna. Theoretical Elasticity. Clarendon Press, Oxford, end ed. edition, 1960.

37. J. G. Simmonds and D. A. Danielson. Nonlinear shell theory with finite rotation and stress-function vectors. J. Appl. Mech., 39:1085-1090, 1972.

38. A. E. Green and P. M. Naghdi. On the derivation of shell theories by direct approach. J. Appl. Mech., March:173-176, 1974.

39. J. C. Simo, M. S. Rifai, and D. D. Fox. On a stress resultant geometrically exact shell model. part vi: Conserving algorithms for non-linear dynamics. International Journal for Numerical Methods in Engineering, 34:117-164, 1992.

40. R. A. Toupin. Theories of elasticity with couple-stress. Arch. Rational Mech. Anal., 17:85-112, 1964.

41. F. Boyer, M. Porez, and A. Leroyer. Poincaré-Cosserat equations for the Lighthill three-dimensional large amplitude elongated body theory: Application to robotics. Journal of Nonlinear Science, 20:47-79, 2010.

42. F. Boyer, M. Porez, A. Leroyer, and M. Visonneau. Fast dynamics of an eel-like robot-Comparisons with Navier-Stokes simulations. Robotics, IEEE Transactions on, 24(6):1274-1288, Dec. 2008.

43. G. D. Weymouth and M. S. Triantafyllou. Ultra-fast escape of a deformable jetpropelled body. Journal of Fluid Mechanics, 721:367 - 385, April 2013.

44. L. M. Milne-Thomson. Theoretical Hydrodynamics. Macmillan, London, 1938. 